\title{
Tails and bridges in the parabolic restricted three-body problem
}

\author{
Esther Barrabés, ${ }^{1}$ Josep M. Cors, ${ }^{2}$ Laura Garcia-Taberner ${ }^{1 \star}$ and Mercè Ollé ${ }^{3}$ \\ ${ }^{1}$ Universitat de Girona, Escola Politècnica Superior, E-17071 Girona, Spain \\ ${ }^{2}$ Universitat Politècnica de Catalunya, Escola Politècnica Superior d'Enginyeria de Manresa, E-08242 Manresa, Spain \\ ${ }^{3}$ Universitat Politècnica de Catalunya, Escola Tècnica Superior d'Enginyeria Industrial de Barcelona, E-08028 Barcelona, Spain
}

Accepted 2017 August 1. Received 2017 July 31; in original form 2017 May 18

\begin{abstract}
After a close encounter of two galaxies, bridges and tails can be seen between or around them. A bridge would be a spiral arm between a galaxy and its companion, whereas a tail would correspond to a long and curving set of debris escaping from the galaxy. The goal of this paper is to present a mechanism, applying techniques of dynamical systems theory, that explains the formation of tails and bridges between galaxies in a simple model, the so-called parabolic restricted three-body problem, i.e. we study the motion of a particle under the gravitational influence of two primaries describing parabolic orbits. The equilibrium points and the final evolutions in this problem are recalled,and we show that the invariant manifolds of the collinear equilibrium points and the ones of the collision manifold explain the formation of bridges and tails. Massive numerical simulations are carried out and their application to recover previous results are also analysed.
\end{abstract}

Key words: methods: numerical-celestial mechanics-galaxies: interactions.

\section{INTRODUCTION}

Gaia data release 1 has reported very recently the discovery of tails around the Large and Small Magellanic Clouds (a pair of massive dwarf galaxies) as well as an almost continuous stellar bridge between them (see Belokurov et al. 2017). Actually, in the seventies, the observation of tails and bridges in multiple galaxies was already recorded. We mention the interacting pairs M51 and NGC 5195 or the pair of interconnected galaxies Arp 295 as two particular examples (see Toomre \& Toomre 1972 and references therein). These papers argue that tails and bridges are just tidal relics of close encounters between two galaxies. In order to study the effects of the brief but violent tidal forces due to a close encounter between the galaxies, several authors have considered a very simple model: each encounter involves only two galaxies assumed to describe parabolic orbits, and each galaxy is idealized as just a disc of non-interacting test particles that initially orbit a central mass point. This model corresponds to the parabolic restricted three-body problem (the parabolic problem along the paper), assuming that the two-point primaries are the galaxies describing parabolic orbits around their common centre of mass.

There are several studies of the observable bridges and tails in galaxies. For instance, Condon et al. (1993) show that galaxies UGC 12914 and UGC 12915 have a continuum bridge that is thought to be due to the collision of the galaxies $2 \times 10^{7}$ years ago, considering that the orbits are nearly parabolic. In Günthardt et al. (2006), the authors consider the system AM1003-435 that is composed by two interacting galaxies. They studied the dynamical evolution of the encounter between the galaxies to conclude that they were moving in parabolic orbits. The $N$-body simulation of the orbits of stars in the galaxies shows bridges and tails. Also using the parabolic model, Namboodiri, Kochhar \& Alladin (1987) studied the existence of bridges and tails in interacting galaxies depending on the circular velocity of the stars within the galaxies.

The parabolic model has also been used in the study of close encounters between disc-surrounded stars and the formation of planets. Pfalzner et al. (2005) studied the change of mass between stars when one or both of them are surrounded by a disc of low-mass particles. They concluded that, in the coplanar case, there were more change of particles between stars when the encounter was prograde. Fragner \& Nelson (2009) studied the effect of parabolic encounters in the formation of Jovian-mass planets. They concluded that planets that have been formed after encounters are more massive and also have greater semimajor axes. Steinhausen, Olczak \& Pfalzner (2012) studied the influence on the initial density of the particles in the change of mass between star-disc encounters, concluding that the shape of the mass distribution has a high effect on the final outcome. Finally, Faintich (1972) considered a Sun-star-comet system to determine the effect of the stellar encounter on the trajectory of the comet but considering a hyperbolic model instead of a parabolic one.

The goal of this paper is, applying techniques of dynamical systems theory, to describe a mechanism that explains the formation of bridges and tails in the very simple model of the parabolic problem. Without trying to make a definition, a bridge would be an arm 
between the two galaxies, whereas a tail would correspond to a long and curving set of particles escaping from a galaxy. More precisely, along the paper, we show that the invariant manifolds of the equilibrium points of the parabolic problem and those of the equilibrium points inside the collision manifolds are the clue to find out such mechanism.

We do massive numerical simulations, considering both equal and unequal primaries, and we show the unambiguous appearance of bridges and tails, after the close encounter of the primaries. In particular, inspired in Toomre \& Toomre (1972), we repeat some of their computations and conclude that our mechanism applies to their simulations (although in that paper, no mention of dynamical systems tools is done at all).

The application of these tools is well known in Celestial Mechanics. However, as far as we know, the application of invariant manifolds to study the close approach of two galaxies in parabolic orbits is a novelty introduced in Alvarez, Cors \& Delgado (2006) and Barrabés, Cors \& Olle (2015). It is worth mentioning that there exist several papers where invariant manifolds have been applied in galactic dynamics. In a germinal paper by Romero-Gómez et al. (2006), the authors introduce a new theory for the formation of ring structures in barred galaxies. Concretely, they propose that rings are formed by material from the invariant manifolds associated with a type of periodic orbits. A study of building blocks and the morphology of rings and spirals in barred galaxies can be found in Athanassoula, Romero-Gómez \& Masdemont (2009a,b). See also Tsoutsis et al. (2009) and the references therein.

In Barrabés et al. (2015), we studied the main features of the parabolic problem only for the case of equal primaries. In the present paper, we consider the parabolic problem for any value of the mass parameter $\mu$, where $1-\mu$, and $\mu$, for $\mu \in(0,1 / 2]$, are the masses of the two primaries in normalized units. First, we show the main features of the problem and secondly, we show the mechanism that explains the formation of tails and bridges.

More concretely, in Section 2, we describe the parabolic problem and the main relevant properties. The parabolic problem is gradientlike due to the existence of a piecewise monotone function, called Jacobi function. This property allows to classify all possible final evolutions on the dynamics of the parabolic problem. On one hand, the flow of the system is extended when the primaries are at infinity, so the phase space is compactified in the time variable and we obtain what we call the global system. The equilibrium points of the global system and their invariant manifolds will be some of the main actors in the description of the dynamics of the problem. In particular, we show that the invariant manifolds of codimension 1 play a key role because they separate the different types of orbits, i.e. they act as frontiers and divide the phase space in regions where the final evolution is either capture or escape. On the other hand, since we are interested in solutions that have close paths to the primaries (or even collide with them), the regularization of the equations in synodical coordinates is also performed (as far as the authors know, no paper dealing with the parabolic problem has ever considered regularized equations). There are two collision manifolds that correspond to a collision between the particle and each primary. The associated stable/unstable invariant manifolds will be the remaining cast of actors in the paper.

Section 3 is devoted to show that the stable invariant manifolds associated with the collinear equilibrium points and the unstable manifold associated with the equilibrium points in the collision manifolds are responsible for the existence of bridges and tails. The results are exemplified by some numerical explorations.
In the Discussion section, some results from Toomre \& Toomre (1972) are recovered and compared with the ones obtained by the authors. Finally we draw some conclusions.

\section{DESCRIPTION OF THE MODEL AND MAIN FEATURES}

In order to describe the dynamical mechanisms that can help understand the formation of bridges and tails when two galaxies have a close encounter, we consider a model already used by Toomre and Toomre. On one hand, each galaxy is modelled as a disc of noninteracting particles orbiting around a central mass point, whereas the two mass points move in parabolic orbits with respect to their centre of mass. Therefore, our simulations are based on a parabolic restricted three-body problem with a set of massless particles. The model is rather simple and does not pretend to simulate the complicated inner dynamics of the galaxies. But although its simplicity, this academic model is good enough to give a dynamical explanation of the formation of bridges and tails that can be used as a basis to understand more complicated models.

In this section, we present briefly the equations of the motion of the model, called parabolic problem, and other main features. The details can be found in Alvarez et al. (2006) and Barrabés et al. (2015).

\subsection{Equations of motion}

Let $m_{1}$ and $m_{2}$ be two bodies, called primaries, moving in parabolic orbits around their common centre of mass. Consider a third body with infinitesimal mass moving under the gravitational attraction of the primaries in the same plane of the motion without affecting them. The planar parabolic restricted three-body problem (simply parabolic problem along the paper) describes the motion of the infinitesimal mass.

We can consider, without loss of generality, suitable units of mass, length and time, such that the constant of gravitation is equal to 1 and, the masses of the primaries are $m_{1}=1-\mu$ and $m_{2}=\mu, \mu \in$ $(0,0.5]$, called the mass parameter. Without any other interaction, when the primaries move in a parabolic motion, the relative position vector from $m_{2}$ to $m_{1}$ can be written as

$\boldsymbol{R}=\left(\sigma^{2}-1,2 \sigma\right), \quad \sigma=\tan (f / 2)$,

where the angle $f$ is known as the true anomaly, and it is measured in the direction in which the relative position is described, starting from pericentre (see e.g. Danby 1992). Note that with the normalized units, the minimum distance between the primaries (when $f=0$ ) is equal to 1 . In this case, the Kepler's equation writes $t-T=$ $\sqrt{2}\left(\sigma+\sigma^{3} / 3\right)$, where $T$ is the time of passage at the pericentre of the parabolic orbits. Without loss of generality, we can take $T=0$; see Fig. 1.

Then, the equation of the motion of an infinitesimal mass moving under the gravitational attraction of the two-point masses in an inertial system of coordinates $Z=(X, Y)$, with origin located at the centre of mass of the primaries, is given by

$\ddot{\boldsymbol{Z}}=-(1-\mu) \frac{\boldsymbol{Z}-\boldsymbol{Z}_{1}}{\left|\boldsymbol{Z}-\boldsymbol{Z}_{1}\right|^{3}}-\mu \frac{\boldsymbol{Z}-\boldsymbol{Z}_{2}}{\left|\boldsymbol{Z}-\boldsymbol{Z}_{2}\right|^{3}}$,

where $=\mathrm{d} / \mathrm{d} t, \boldsymbol{Z}_{1}=\boldsymbol{Z}_{1}(t)=\mu \boldsymbol{R}$ and $\boldsymbol{Z}_{2}=\boldsymbol{Z}_{2}(t)=(\mu-1) \boldsymbol{R}$ are the position vectors of the primaries; see Fig. 1. 


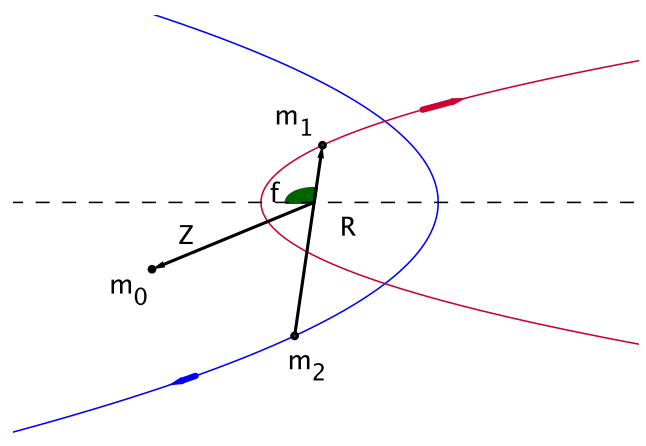

Figure 1. Parabolic problem. The two main bodies $m_{1}$ and $m_{2}$ move in parabolic orbits, where $\boldsymbol{R}$ is their relative position and the angle $f$ is known as the true anomaly. This restricted three-body model describes the motion of any massless particle $m_{0}$ under their gravitational attraction.

First, we introduce a rotating coordinate system $z=(x, y)$ where the primaries remain fixed along the new $x$-axis at $z_{1}=(\mu, 0)$ and $z_{2}=(\mu-1,0)$. The change is given by the complex product

$\boldsymbol{Z}=\boldsymbol{R} z$.

Secondly, a change of time via $\frac{\mathrm{d} t}{\mathrm{~d} s}=\sqrt{2} R^{3 / 2}$, where $R=|\boldsymbol{R}|$ is introduced. The variable $\sigma$ can be expressed in terms of the new time $s$ as $\sigma=\sinh (s)$. Note that the primaries tend to infinity along their parabolic orbits, i.e. $R \rightarrow \infty$ when $t \rightarrow \pm \infty$, or equivalently, when $s$ tends to $\pm \infty$, and that the closest passage takes place at $s=0$. The system obtained with such changes is non-autonomous and nonperiodic, i.e. the vector field depends on non-periodic functions, concretely, on $\tanh (s)$ and $\operatorname{sech}(s)$.

In order to apply the standard tools of dynamical systems, like the study of the existence of equilibrium points or periodic orbits, and their stability, it is more convenient to have an autonomous system, or at least periodic. In this case, we can transform the system to an autonomous one through the change $\sin (\theta)=\tanh (s)$. After straightforward computations, with the new variables $\left(\theta, z, w=z^{\prime}\right)$, the equation (2) becomes the following autonomous system (see Alvarez et al. 2006, for details)

$$
\left\{\begin{aligned}
\theta^{\prime} & =\cos \theta, \\
\boldsymbol{z}^{\prime} & =\boldsymbol{w}, \\
\boldsymbol{w}^{\prime} & =-A(\theta) \boldsymbol{w}+\nabla \Omega(\boldsymbol{z}),
\end{aligned}\right.
$$

where $^{\prime}=\frac{\mathrm{d}}{\mathrm{d} s}$ denotes the derivative with respect to $s$,

$$
\mathbf{A}(\theta)=\left(\begin{array}{cc}
\sin \theta & 4 \cos \theta \\
-4 \cos \theta & \sin \theta
\end{array}\right)
$$

and

$\Omega(z)=x^{2}+y^{2}+\frac{2(1-\mu)}{\sqrt{(x-\mu)^{2}+y^{2}}}+\frac{2 \mu}{\sqrt{(x+1-\mu)^{2}+y^{2}}}$.

The new variable $\theta$ can be interpreted as the time: on one hand, observe that $R=1 / \cos ^{2} \theta$, therefore, each value of $\theta$ provides also the distance between the primaries. On the other hand, when $s \in$ $(-\infty,+\infty), \theta$ varies in $(-\pi / 2, \pi / 2)$, and the limits $s \rightarrow \pm \infty$ correspond to $\theta \rightarrow \pm \pi / 2$. Note that $\theta^{\prime}>0$, so it is an increasing function: when $\theta$ is close to $-\pi / 2$, the primaries are far apart, then they go close, with the minimum distance when $\theta=0$, and separate as they escape through their parabolic orbits when $\theta$ tends to $\pi / 2$. We also remark that, if there exists any equilibrium point (as we will see in the next section), it must satisfy $\theta= \pm \pi / 2$. Mathematically, the system (4) is well defined when $\theta= \pm \pi / 2$ (i.e. when the primaries are 'at infinity'), and the corresponding equations are invariant subsets of the system. We call them upper and lower boundary problem, respectively. The key point to consider these boundary problems is that although the study of the dynamics 'at infinity' has no physical meaning, it will help understand some behaviours when the two primaries go close, as we will show. More precisely, a natural way to start studying a non-linear system of Ordinary Differential Equations (ODE) is by the computation of the simplest solutions, the equilibrium points. We will compute them (at $\theta= \pm \pi / 2$ ) and we will also study their stability, which will give rise to the computation of the associated invariant manifolds that do exist for the whole system 4, also when the primaries are separated by a finite distance. The dynamics of these manifolds in the finite regime will be relevant for the purpose of this paper: the explanation of formation of bridges and tails.

The extended phase space of system (4) is given by $D=$ $[-\pi / 2, \pi / 2] \times\left(\mathbb{R}^{2}-\{(\mu-1,0),(\mu, 0)\}\right) \times \mathbb{R}^{2}$. We will call the system (4) the global system, and we denote as configuration space the projection of $D$ on to the $(x, y)$ plane. Note that equation (4) has two singularities at the (fixed) location of the primaries $z=z_{1}$ and $z=z_{2}$.

Moreover, the problem satisfies the following symmetry:

$\left(s, \theta, x, y, x^{\prime}, y^{\prime}\right) \rightarrow\left(-s,-\theta, x,-y,-x^{\prime}, y^{\prime}\right)$.

The symmetry implies that for any solution of (4), there exists another one symmetric with respect to $y=0$ in the configuration plane reversing time.

\subsection{Equilibrium points, homothetic solutions and the Jacobi function}

All equilibrium points of the global system are points belonging to upper and lower boundary problems, i.e. all of them correspond to $\theta= \pm \pi / 2$. As was shown in Alvarez et al. (2006), the equilibrium points of the parabolic problem in each boundary coincide with the classical five equilibrium points of the circular restricted threebody problem (see Szebehely 1967, for example): three collinear and two triangular. We denote by $L_{i}^{+}$and $L_{i}^{-}, i=1, \ldots, 5$, the equilibrium points for $\theta=\pi / 2$, and $\theta=-\pi / 2$, respectively. Along the paper, and in order to follow the same notation as in Alvarez et al. (2006) and Barrabés et al. (2015), we label the collinear equilibrium points increasingly with respect their location on the $x$-axis: $x\left(L_{1}^{ \pm}\right)<\mu-1<x\left(L_{2}^{ \pm}\right)<\mu<x\left(L_{3}^{ \pm}\right)$; see Fig. 3 .

The study of the stability character of the equilibrium points is achieved through the study of the eigenvalues of the differential matrix of the vector field of (4) at the equilibrium points (see e.g. for a detailed discussion about the study of the linearization of the equations around equilibrium points). In the parabolic problem, all the equilibrium points have associated positive and negative real eigenvalues. This means that there exist unstable (for positive eigenvalues) and stable (for negative eigenvalues) invariant manifolds. The definitions of these manifolds for a generic equilibrium point $L_{\xi}$ are the following. Let be $z(s)$ a solution of (4). Then:

(i) The unstable manifold is the set of orbits that tend to the equilibrium point backwards in time:

$W^{u}\left(L_{\xi}\right)=\left\{z(s) \in \mathbb{R}^{5} / \lim _{s \rightarrow-\infty}\left|z(s)-L_{\xi}\right|=0\right\}$. 
Table 1. Dimension of the invariant manifolds of the equilibrium points in the global system (4).

\begin{tabular}{lcc}
\hline & $L_{1,2,3}^{+}$ & $L_{4,5}^{+}$ \\
\hline $\operatorname{dim}\left(W^{u}\right)$ & 1 & 2 \\
$\operatorname{dim}\left(W^{s}\right)$ & 4 & 3 \\
\hline
\end{tabular}

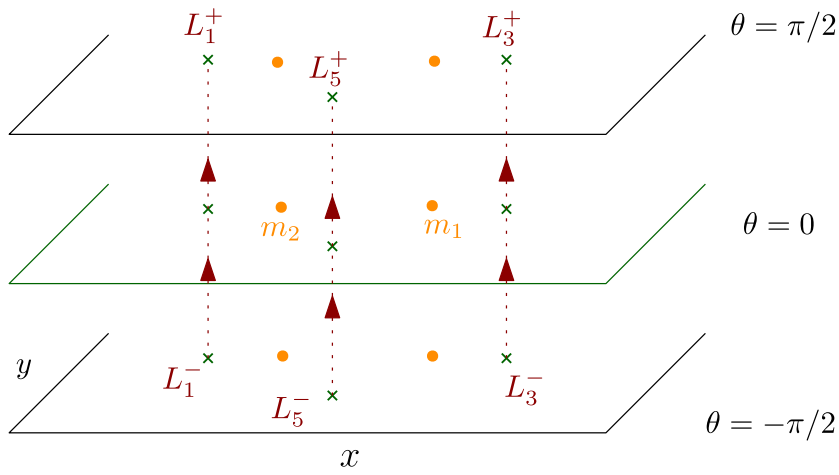

Figure 2. Schematic representation of the homothetic solutions connecting the equilibrium points $L_{i}^{-} \rightarrow L_{i}^{+}, i=1, \ldots, 5$ (only three of them are drawn) in the rotating system. Each plane represents the configuration space $(x, y)$ at each value of $\theta$.

(ii) The stable manifold is the set of orbits that tend to the equilibrium point forwards in time:

$W^{s}\left(L_{\xi}\right)=\left\{z(s) \in \mathbb{R}^{5} / \lim _{s \rightarrow+\infty}\left|z(s)-L_{\xi}\right|=0\right\}$.

The invariant manifolds are invariant subsets of the phase space.

In Alvarez et al. (2006), the dimensions of the invariant manifolds of the equilibrium points were studied. In Table 1, we show the dimensions corresponding to the equilibrium points on the upper boundary problem $L_{i}^{+}, i=1, \ldots, 5$. Using symmetry (7), the dimensions of the invariant manifolds associated with the equilibrium points on the lower boundary problem are obtained. For any collinear equilibrium point $L_{i}^{+}, i=1,2,3$, the unstable manifold is of dimension 1 . That means that $W^{u}\left(L_{i}^{+}\right)$consists in a single orbit. The stable manifold is of dimension 4 , i.e. in the phase space $D$, $W^{s}\left(L_{i}^{+}\right)$consists in a four-dimensional set of orbits that tend to the equilibrium point. This is an important fact: a manifold of dimen- sion $n-1$ (or of codimension 1 ) in a space of dimension $n$ divides the space in subregions. When considering the invariant manifolds associated with the equilibrium points, we will show that $W^{s}\left(L_{i}^{+}\right)$, $i=1,2,3$ separates the phase space in subregions where the orbits have different behaviours, which gives the clue to understand the formation of bridges and tails.

Besides the equilibrium points, the simplest solutions of the global system (4) are the five homothetic solutions connecting the equilibrium points $L_{i}^{-}$with $L_{i}^{+}, i=1 \ldots 5$, and belonging to $W^{u}\left(L_{i}^{-}\right) \cap W^{s}\left(L_{i}^{+}\right)$; see Fig. 2:

$\theta(s)=\arcsin (\tanh (s)), \quad \boldsymbol{z}(s)=\left(x\left(L_{i}^{ \pm}\right), y\left(L_{i}^{ \pm}\right)\right), \quad \boldsymbol{w}(s)=0$.

Clearly, these five homothetic solutions in the sidereal or inertial reference frame are solutions in which the three bodies (the infinitesimal mass and the two primaries) keep the same configuration all the time: either the three bodies lie in a line (collinear configuration) or they lie at the vertices of an equilateral triangle (triangular configuration).

The parabolic problem also admits a function, similar to the Jacobi constant of the circular restricted three-body problem (see Szebehely 1967) that we call, by similarity, the Jacobi function:

$C=2 \Omega(z)-|w|^{2}$.

Unlike the circular problem, $C$ is not constant, in general, along the solutions of the global system (4), but it has a piecewise monotone behaviour along the solutions, except at the homothetic solutions, where its value is constant. Precisely,

$\frac{\mathrm{d} C}{\mathrm{~d} s}=2 \sin \theta|\boldsymbol{w}|^{2}$.

Therefore, along any solution of the global system $C$ decreases when $\theta \in[-\pi / 2,0](s \leq 0)$, whereas for $\theta \in[0, \pi / 2](s \geq 0)$ the function $C$ increases.

Given a value of $C$, let $V_{0}(C)=\{z \mid 2 \Omega(z)=C\}$ be the set called zero velocity curves. Their topology is the same as in the circular restricted three-body problem (see Szebehely 1967). Let $C_{i}=C\left(L_{i}^{ \pm}\right)$, $i=1, \ldots, 5$, be the value of $C$ at each equilibrium point. The socalled Hill's regions, i.e. the allowed regions of motion in the configuration space, for $C$ fixed, can be obtained from (11). We plot in Fig. 3 the zero velocity curves and the forbidden regions of motion (shaded regions) for different fixed values of $C$ with $\mu=0.4$.

We remark the fact that the zero velocity curves change with time: when $s \leq 0$, the curves shrink and Hill's region gets bigger,
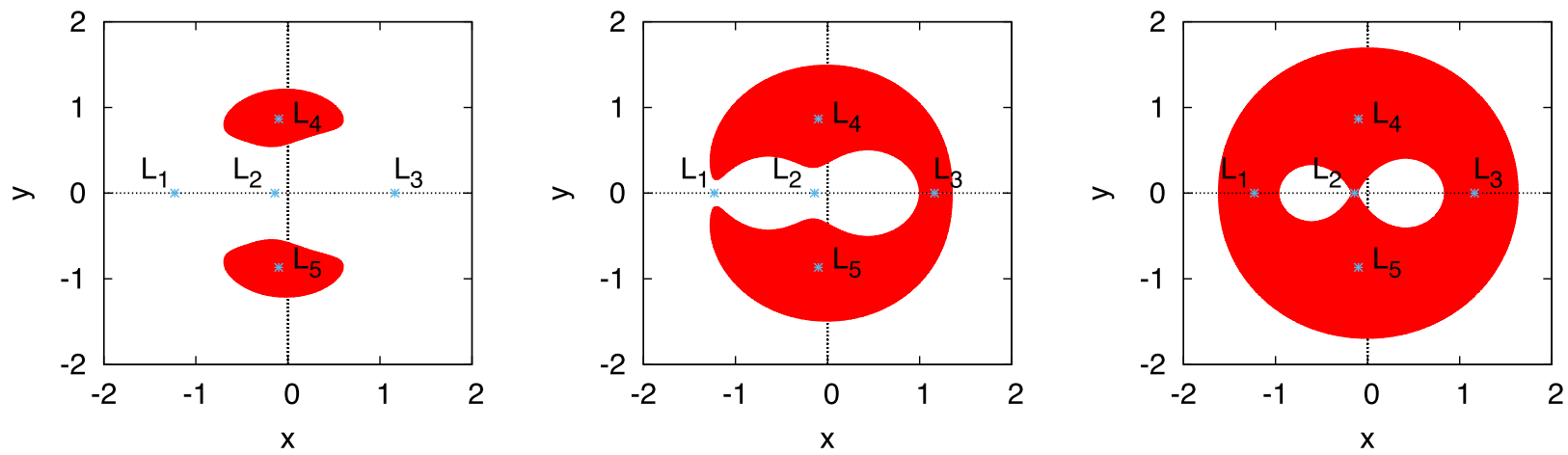

Figure 3. Zero velocity curves and the forbidden (shaded) regions of motion in configuration space for $\mu=0.4$ and the fixed values of the Jacobi function $C=6<C_{3}, C=7 \in\left(C_{3}, C_{1}\right), C=8>C_{2}$ (from left to right). The values of the Jacobi function at the equilibrium points are: $C_{1}=7.0378692601676622$, $C_{2}=7.9618171291425179$ and $C_{3}=6.7581533072377464$. The location of the equilibrium points (although they do not live at the same level of $C$ ) is also shown. 
whereas for $s \geq 0$, the curves get bigger and Hill's region decreases. This is a key factor for the description of the final evolutions of the solutions.

\subsection{Final evolutions}

We want to describe all the possible behaviours of the solutions of system (4) as time tends to infinity. We call them the final evolutions of a trajectory: future evolution, when time tends to $+\infty$ (forwards) and past evolution, when time tends to $-\infty$ (backwards). Note that, using the symmetry (7), for any kind of future evolution, there exists a trajectory exhibiting the same kind of evolution backwards in time. Therefore, the same kind of behaviours are obtained both for past and future. We use in the present paper some results given in Barrabés et al. (2015) for $\mu=1 / 2$. The results are valid for any value of $\mu$, and the proofs are similar, so we do not repeat them here.

We will describe the final evolutions in general (forwards and backwards). Due to the geometry of the zero velocity curves and the nature of the Jacobi function, the final evolutions are rather simple, mainly, escape and capture orbits. Essentially, an escape orbit is a trajectory that goes far apart from both primaries; a capture orbit is a trajectory that gets trapped around one primary (spinning around it) or even collides with the primary. More specifically, when $s \rightarrow$ $\infty$ (representing indistinctly $\pm \infty$ ) any solution of system (4) can be classified as:

(i) Capture orbit. It is an orbit that the synodical distance to one of the primaries tends to zero as time tends to infinity:

$\lim _{s \rightarrow \infty}\left|z(s)-z_{j}\right|=0$,

where $j$ can be 1 or 2 . That means that the massless particle approaches one of the primaries and keeps around it as time increases. Eventually, the particle can also collide with the primary (recall the singularities of the equations).

All of the capture orbits tend to a collision with one of the primaries (in general at infinity time). Analogously to the definitions of the invariant manifolds associated with an equilibrium point, the set of all trajectories ending in a collision with the corresponding primary forwards/backwards in time will be denoted by $W^{s}\left(m_{j}\right)$ or $W^{u}\left(m_{j}\right), j=1,2$, respectively. They are called the stable/unstable manifolds associated with collision manifold.

(ii) Escape orbit. It is an orbit that keeps away from any primary as time tends to infinity:

$\lim _{s \rightarrow \infty}\left|\boldsymbol{z}(s)-\boldsymbol{z}_{j}\right|>\delta$,

for a fixed value of $\delta$. Using (3), this means that the inertial distance $\left|\boldsymbol{Z}(t)-\boldsymbol{Z}_{j}(t)\right| \rightarrow \infty$ as $t \rightarrow \infty$ for $j=1,2$, so the particle escapes from any neighbourhood of any primary.

Note that, regarding future evolutions, the orbits belonging to $W^{s}\left(L_{i}^{+}\right), i=1, \ldots, 5$, satisfy the escape condition. By definition, an orbit on the stable manifold of any equilibrium point $L_{i}^{+}$will tend to that point as $s \rightarrow+\infty$. In particular, it does not approach any primary. We want to differentiate these orbits from the orbits that escape without any particular configuration. Focusing on future evolutions:

(a) an orbit escapes in collinear configuration with the primaries if it belongs to $W^{s}\left(L_{i}^{+}\right), i=1,2,3$;

(b) an orbit escapes in equilateral triangular configuration with the primaries if it belongs to $W^{s}\left(L_{i}^{+}\right), i=4,5$;

(c) an orbit escapes with no configuration, otherwise.
Table 2. Position of equilibrium points $L_{2}^{ \pm}$and the value of $C_{2}=C\left(L_{2}^{ \pm}\right)$ for three different values of $\mu$.

\begin{tabular}{lccc}
\hline & $\mu=0.5$ & $\mu=0.3$ & $\mu=0.1$ \\
\hline$L_{2}^{ \pm}$ & $(0,0)$ & $(-0.2861297821,0)$ & $(-0.6090351100,0)$ \\
$C_{2}=C\left(L_{2}^{ \pm}\right)$ & 8 & 7.840299166 & 788.193906458 \\
\hline
\end{tabular}

The same definitions can be done for past evolutions and $W^{u}\left(L_{i}^{-}\right)$, $i=1, \ldots, 5$.

Any trajectory can be classified depending on their past and future evolution (which, of course, may be different). The evolution of Hill's regions (again, forwards and backwards in time) allows us to obtain a criterion to classify the orbits depending on their final evolution. We state the criterion for future evolutions, clearly the similar criterion can be obtained for past evolutions.

\section{C-criterion}

Let $\mathbf{q} \in \operatorname{Int}(D)$ with $\theta \geq 0$, and $\gamma(s)=(\theta(s), z(s), \boldsymbol{w}(s)), s \in[0$, $\infty$ ), the solution of the global system (4) through q. Then,

(i) if for some time $s_{0}$ the value of the Jacobi function $C\left(\gamma\left(s_{0}\right)\right)>C_{2}$ and $z(s)$ is located in one of the bounded components of Hill's region, then it is a collision orbit (see Fig. 3 right);

(ii) if for some time $s_{0}$ the value of the Jacobi function $C\left(\gamma\left(s_{0}\right)\right)>C_{1}$ and $z(s)$ is located in the unbounded component of Hill's region, then the orbit escapes with no configuration (see Fig. 3 centre and right).

For all values of $\mu, C_{1}<C_{2}$. Therefore, for future evolutions, it is sufficient to monitor if $C(\gamma(s))>C_{2}$ to be able to classify the orbit. In Table 2, we show the value of $C_{2}$ for the values of $\mu$ for which we will present some results in Section 3.

\subsection{Regularization of the equations of motion}

In order to study the collision with a primary and to deal numerically with orbits going close to the primaries, we need to remove the singularities $r_{i}=0, i=1,2$ appearing in the equations of motion (4). In order to do so, we follow McGehee's ideas (see Pinyol 1995 for the elliptic Restricted Three Body Problem (RTBP) and references therein) to obtain the so-called regularized system of equations.

The regularization removes one singularity, $r_{1}=0$ or $r_{2}=0$ at a time. We describe here the procedure to remove the collision with $m_{1}$. We perform the following changes of variables:

(i) We move the selected primary $m_{1}$ to the origin:

$\left(x, y, x^{\prime}, y^{\prime}, \theta\right) \rightarrow\left(X_{1}, X_{2}, X_{3}, X_{4}, \theta\right)$

with $x=X_{1}+\mu, y=X_{2}, x^{\prime}=X_{3}$ and $y^{\prime}=X_{4}$.

(ii) We proceed now with polar coordinates

$\left(X_{1}, X_{2}, X_{3}, X_{4}, \theta\right) \rightarrow(r, \delta, \bar{y}, \bar{x}, \theta)$

with $X_{1}=r \cos \delta, X_{2}=r \sin \delta, \bar{y}=r^{\prime}, \bar{x}=r \delta^{\prime}$.

(iii) We now consider

$(r, \delta, \bar{y}, \bar{x}, \theta) \rightarrow(r, \delta, v, u, \theta)$

with $v=\sqrt{r} \bar{y}, u=\sqrt{r} \bar{x}$.

(iv) Finally, we introduce a new time variable $\tau$ by $\frac{\mathrm{d} s}{\mathrm{~d} \tau}=r^{3 / 2}$. 
After the computations to implement the above changes of variables and time, system (4) becomes

$$
\left\{\begin{aligned}
& \dot{r}=v r, \\
& \dot{\delta}=u, \\
& \dot{v}= \frac{1}{2} v^{2}+u^{2}-(v \sin \theta+4 u \cos \theta) r^{3 / 2}+2 r^{3}+2 \mu r^{2} \cos \delta \\
& \quad-2(1-\mu)-\frac{2 \mu r^{2}}{r_{2}^{3}}(r+\cos \delta), \\
& \dot{u}=-\frac{1}{2} u v+(4 v \cos \theta-u \sin \theta) r^{3 / 2}+2 \mu r^{2} \sin \delta\left(-1+\frac{1}{r_{2}^{3}}\right), \\
& \dot{\theta}=r^{3 / 2} \cos \theta,
\end{aligned}\right.
$$

where $=\mathrm{d} / \mathrm{d} \tau$ and $r_{2}=\sqrt{r^{2}+2 r \cos \delta+1}$. We notice that this new system has only the singularity $r_{2}=0$.

We proceed in a similar way to remove the singularity $r_{2}=0$ from system (4), so the new associated system has only the singularity $r_{1}=0$. Along the integration of any trajectory, for a given initial condition, we integrate numerically system (4) unless the particle is in a neighbourhood of one of the primaries, where we apply the changes of variables and time and integrate the corresponding regularized system of equations.

\subsection{Dynamics of the parabolic problem}

The dynamics of the parabolic problem can be understood focusing on its future and past evolutions and looking for the heteroclinic connections, i.e. orbits that connect two invariant objects, such that, two equilibrium points (as the homothetic solutions) or a primary and an equilibrium point (therefore, an orbit belonging to $W^{u}\left(m_{j}\right)$ and $W^{s}\left(L_{i}^{+}\right)$). See Barrabés et al. (2015) for details.

The existence of few different types of final evolutions becomes enriched due to existence of the homothetic solutions. Since they belong to $W^{u}\left(L_{i}^{-}\right) \cap W^{s}\left(L_{i}^{+}\right)$, they are a natural way to transport the dynamics near the lower boundary problem to the upper one (see Fig. 2). Moreover, in the case of the collinear equilibrium points, the invariant manifolds of codimension 1 (the orbits that escape, forwards or backwards in time, with linear configuration) behave as a frontier and divide the phase space in regions of capture or escape with no configuration. We will see in Section 3 that these frontiers are also responsible for the existence of bridges and tails.

In order to emphasize the importance of the invariant manifolds of codimension 1 associated with the collinear equilibrium points, and how they separate the different type of orbits, we will reproduce some results from Section 4.2 in Barrabés et al. (2015) $(\mu=0.5$ in that paper) for values of $\mu \neq 0.5$. More precisely, we take initial conditions at $\theta=0$ in the plane $\left(x, y^{\prime}\right)$, i.e. with $y=x^{\prime}=0$. These orbits are symmetric with respect to $\theta=0$, so they have the same final evolution forwards and backwards in time. We integrate these initial conditions forward in time and classify the orbits using the $C$-criterion. Note that due to the fact that some orbits can have a close encounter to a primary, a binary collision regularization is performed in order to continue the integration.

In Fig. 4, the detailed regions of escape with no configuration (in white) and capture around $m_{1}$ (in red) or around $m_{2}$ (in blue), on the $\left(x, y^{\prime}\right)$ plane, are shown for different values of $\mu$.

Clearly, according to the possible final evolutions described in Subsection 2.3, the frontiers between escape with no configuration and capture regions must correspond to orbits that escape with a certain configuration, i.e. they belong to $W^{s}\left(L_{i}^{+}\right)$for a certain equilibrium point. As mentioned, the only stable manifolds of codi- mension 1 are those of $L_{i}^{+}, i=1,2,3$. The frontier between two regions of capture orbits around different primaries corresponds to $W^{s}\left(L_{2}^{+}\right)$, between an escape with no configuration region and a capture around $m_{1}$ is $W^{s}\left(L_{3}^{+}\right)$and between an escape region with no configuration and a capture around $m_{2}$ is $W^{s}\left(L_{1}^{+}\right)$.

To illustrate that, we consider, for $\mu=0.3$, the region in the $\left(x, y^{\prime}\right)$ plane $[-1.05,-0.9] \times[-4.1,-3.8]$, and a certain number of initial conditions $\left(x, y=0, x^{\prime}=0, y^{\prime}\right)$ in a circle of radius 0.05 around the point $x=-0.96$ and $y^{\prime}=-3.88$, labelled as a,...,h; see Fig. 5 left. The circle contains initial conditions from the three regions (capture around $m_{1}$, capture around $m_{2}$ and escape with no configuration). In Fig. 5 right, we show the evolution of the corresponding orbits (each label on the left plot corresponds to initial conditions of the orbit with the same label on the right plot): orbit $a$ escapes with no configuration, whereas orbit $b$ is captured by $m_{1}$. Therefore, by continuity there must be one orbit tending to $L_{3}^{+}$. That is, the frontier between white and red regions must be orbits belonging to $W^{s}\left(L_{3}^{+}\right)$. Analogously, orbits $c$ and $d$ are captured by $m_{1}$ whereas orbit $e$ is captured by $m_{2}$. Again by continuity there exists an orbit that tends to $L_{2}^{+}$. Thus, the frontier between red and blue regions must be orbits belonging to $W^{s}\left(L_{2}^{+}\right)$. The same reasoning can be done with orbits $f$ (capture $m_{2}$ ) and $g$ (escape with no configuration): in between the frontier corresponds to $W^{s}\left(L_{1}^{+}\right)$.

From now on, the orbits that escape with no configurations will be simply called escape orbits.

\section{GENERATING BRIDGES AND TAILS}

Our goal in this section is to show that the stable invariant manifolds associated with the collinear equilibrium points, $W^{s}\left(L_{i}^{+}\right), i=1,2$, 3 , and the unstable invariant manifold associated with the collision with one primary, $W^{u}\left(m_{i}\right), i=1,2$, are responsible for the existence of bridges and tails. We perform a general and broad exploration considering big sets of initial conditions around a primary and we classify them depending on the final evolution forwards in time after the close encounter of the primaries using the $C$-criterion. We show and comment the results obtained for $\mu=0.5$, and then for $\mu \neq 0.5$.

The general exploration is performed as follows. First, we fix a negative value of $\theta=\theta_{0}$ (a time before the close encounter) and a value of $C \geq C_{2}$. For this value of $C$, we know that Hill's region has a bounded component around $m_{j}, j=1,2$ (see Fig. 3). Next, we fix a primary, $m_{j}$, and take a circle centred at that primary and radius $r_{c}$ such that it is contained in the bounded component of Hill's region. Then, we generate a set of initial conditions $\left(x, y, x^{\prime}, y^{\prime}\right)$ around the primary $m_{j}$ as:

$$
\begin{array}{llrl}
x & =x_{m_{j}}+r_{c} \cos \alpha & x^{\prime} & =v \cos \beta \\
y & =r_{c} \sin \alpha & y^{\prime} & =v \sin \beta,
\end{array}
$$

where $x_{m_{1}}=\mu$ and $x_{m_{2}}=\mu-1, \alpha, \beta \in[0,2 \pi]$ and $v$ is obtained from (11). For any given $\alpha$ and $\beta$, we take the corresponding initial condition and we follow its trajectory, forward in time. Applying the $C$-criterion, each trajectory will be classified as a collision orbit with one of the primaries or an escape orbit.

For each fixed value of $C$ and $r_{c}$, we take $N$ equally spaced values of $\alpha$ and $\beta$. We show the results in two different ways:

(i) Classification plots. We plot in the $(\alpha, \beta)$ plane each point in different colours depending on the final evolution: red (light grey in black/white printed version) - captured by $m_{1}$, blue (dark grey in black/white printed version) - captured by $m_{2}$ or white (blank) escape. 

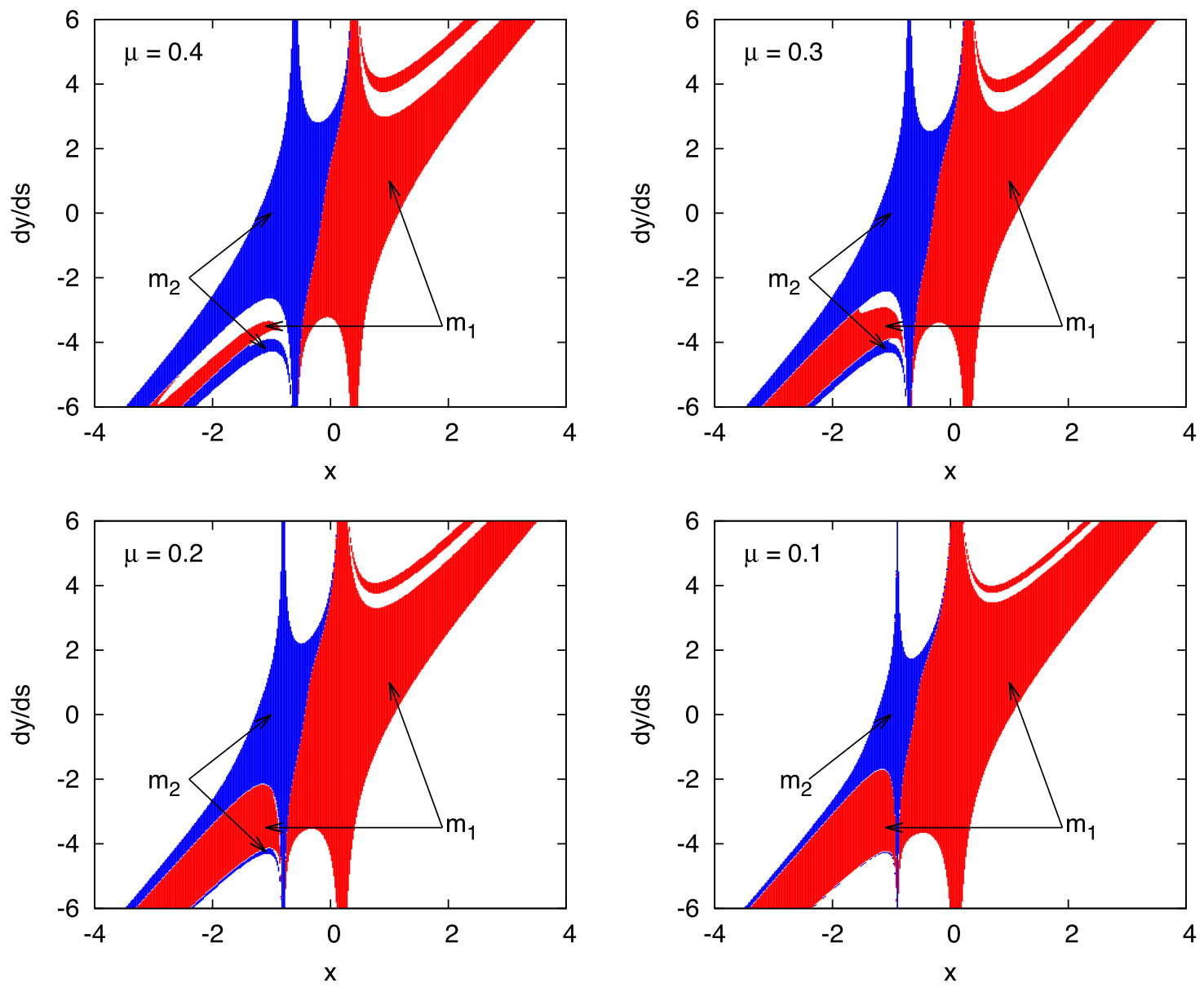

Figure 4. Regions of escape with no configuration (white regions) and capture around $m_{1}$ (red) and $m_{2}$ (blue) in the $\left(x, y^{\prime}\right)$ plane for $\mu=0.4,0.3,0.2,0.1$ from top to bottom, left to right.
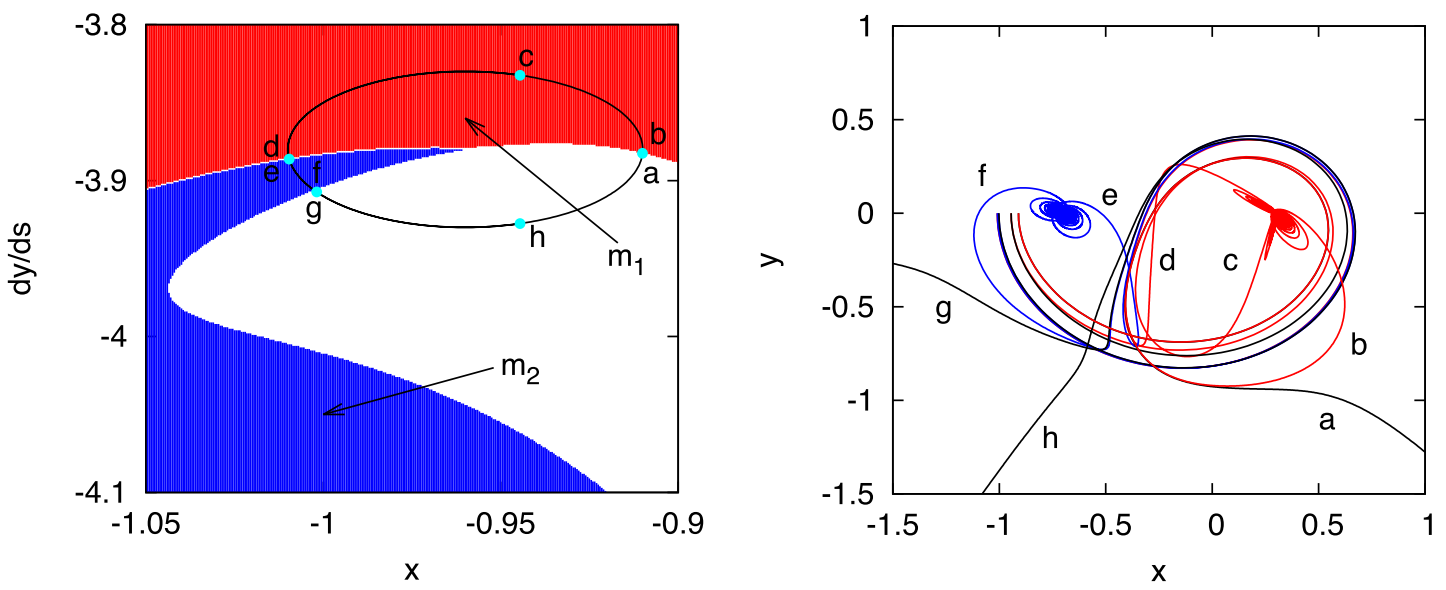

Figure 5. The left plot shows a magnification of the regions of capture around $m_{1}$ (red) and $m_{2}$ (blue) for $\mu=0.3$. On the right plot, there are different orbits that escape, tend to $m_{1}$ or tend to $m_{2}$.

(ii) Snapshots. We plot the location of the particles in the configuration inertial frame $(X, Y)$ for different values of time $\theta>0$. The code of colours red and blue is the same one. The orbits that escape are plotted in black in these figures.

We will see how, considering different regions in the classification plots (and also for different values of $r_{c}$ and $C$ ), the snapshots show the existence of bridges and tails.
Several comments regarding such initial conditions and the classification should be made. We observe that any negative value of $\theta_{0}$ might be taken. The closer the value of $\theta_{0}$ to zero, the smaller the distance between the primaries. We do not want to start too far from the closest passage between the primaries, nor too close. Moreover, any particle at a distance $r_{c}$ of the primary in synodical coordinates is at a distance $R_{c}=r_{c} R$ of that primary in the inertial frame, where $R=1 / \cos ^{2} \theta_{0}$ is the inertial distance between the primaries. Thus, 

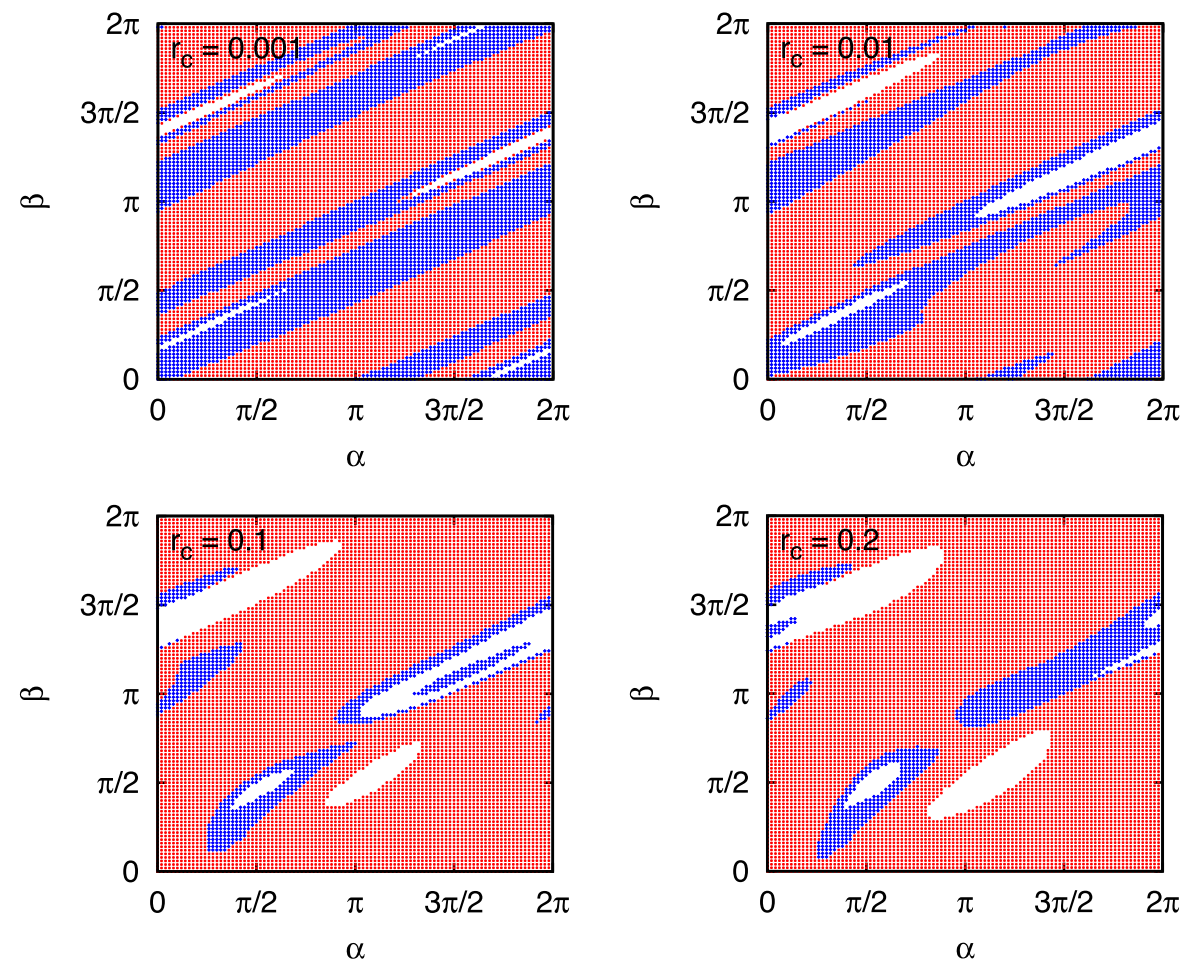

Figure 6. Classification of the final evolutions of the initial conditions (19) in the ( $\alpha, \beta)$ plane for $C=8$ and $r_{c}=0.001,0.01,0.1,0.2$ from top to bottom, left and right. Red and blue (light and dark grey, respectively) correspond to orbits tending to $m_{1}$ and $m_{2}$, respectively. White corresponds to orbits that escape $(\mu=0.5)$.

$r_{c}$ represents the ratio between the distance of the particle to the primary and the distance between the two primaries. We consider $\theta_{0}=-\pi / 4$, so $R=2$, and $r_{c} \in(0,0.2]$. Then, we have a set of initial conditions that forms an annulus that spreads around the primary up to 20 per cent the distance between the two primaries in the inertial frame.

We also remark that the strategy to take such initial conditions inside the bounded component of neighbourhood Hill's region guarantees, using the $C$-criterion for past evolutions, that all the trajectories considered tend to collision with $m_{j}$ backwards in time. That is, all the trajectories considered belong to the unstable manifold of the collision with $m_{j}, W^{u}\left(m_{j}\right)$.

\subsection{Results for equal masses}

We consider the case $\mu=0.5$ that corresponds to equal masses of both primaries. We take a set of test particles around one primary as in (19). Since both primaries have the same mass, it is enough to do the exploration only for one primary. We consider in this section all the particles leaving a neighbourhood of $m_{1}$.

In Fig. 6, we show the classification plots for $C=8$ and several values of $r_{c}$. We have taken $N=100$ (bigger values of $N$ convey only to the same figures with more precision but the same information). As mentioned before, all the orbits belong to $W^{u}\left(m_{1}\right)$ (capture backwards in time), but forwards in time, different behaviours appear clearly: there exist orbits captured by $m_{1}$ or $m_{2}$ or escaping (red, blue and white colours, respectively).

When $r_{c}$ is small, we observe that there are few orbits that escape, a big percentage of orbits that remain captured by $m_{1}$ and a significant percentage of orbits that jump and become captured by the other primary $m_{2}$. However, when $r_{c}$ increases, the number of orbits that escape also increases but the number of jumping orbits to the other primary decreases. We also observe that the different coloured regions vary with $r_{c}$ but there is not a clear structure of how such regions evolve. Similar patterns and results are obtained for different values of $C$ : in general, we find orbits tending to any of the primaries or escaping, except if $r_{c}$ is too small. The bigger the value of $C$, the smaller the Hill's region around the primaries, and the less the probability to escape.

We now focus on the frontier separating the different coloured regions. In Section 2.6, we showed that the stable manifolds of $L_{i}^{+}$, $i=1,2,3$ separate different types of orbits and the points on the boundary between different coloured regions (see Figs 4 and 5) precisely belong to $W^{s}\left(L_{i}^{+}\right)$for a suitable $i=1,2,3$. In a similar way now, the points on the frontier separating the different coloured regions that belong to $W^{u}\left(m_{1}\right)$ also belong to:

(i) $W^{s}\left(L_{1}^{+}\right)$if the boundary curve separates an escape region (white) from a region of collision to $m_{2}$ (blue);

(ii) $W^{s}\left(L_{3}^{+}\right)$if the boundary curve separates an escape region (white) from a region of collision to $m_{1}$ (red);

(iii) $W^{s}\left(L_{2}^{+}\right)$if the boundary curve separates two different regions of collision (blue and red).

Therefore, the curves that separate two different regions belong to $W^{s}\left(L_{i}^{+}\right) \cap W^{u}\left(m_{1}\right)$, i.e. they are heteroclinic orbits connecting a collision with $m_{1}$ and an equilibrium point.

Next, we show that these heteroclinic orbits provide simple mechanisms to explain tails and bridges. To do so, let us focus on selected ranges in the $(\alpha, \beta)$ plane where two different coloured regions appear. We choose two zones: a red region (collision to $m_{1}$ ) surrounding a white one (escape region) (see the box in Fig. 7, left), and a blue region (collision to $m_{2}$ ) surrounded by a red one (collision to $m_{1}$ ) (see the box in Fig. 8, left). We also compute, using bisection method, the points on the frontier (thick black points).

Regarding the first zone, we focus on the separation points between escape and capture with $m_{1}$ - the black curve in Fig. 7, 

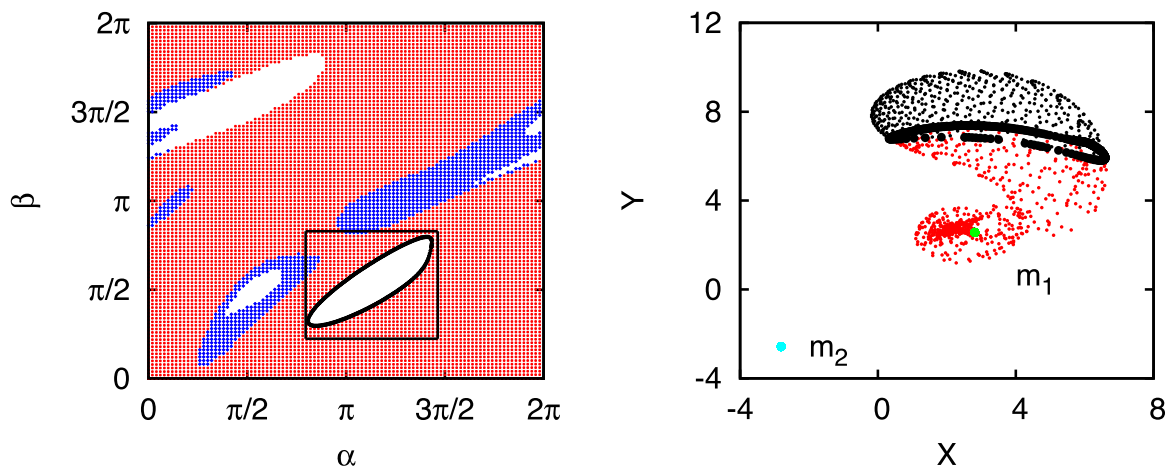

Figure 7. Left: classification plot of $(\alpha, \beta)$ points for $C=8$ and $r_{c}=0.2$. The rectangle shows the region where the initial conditions are taken (only points in the red and white regions). Right: snapshot for $\theta=1.2$. Points on the $W^{s}\left(L_{3}^{+}\right)$(thick black) are also plotted $(\mu=0.5)$.
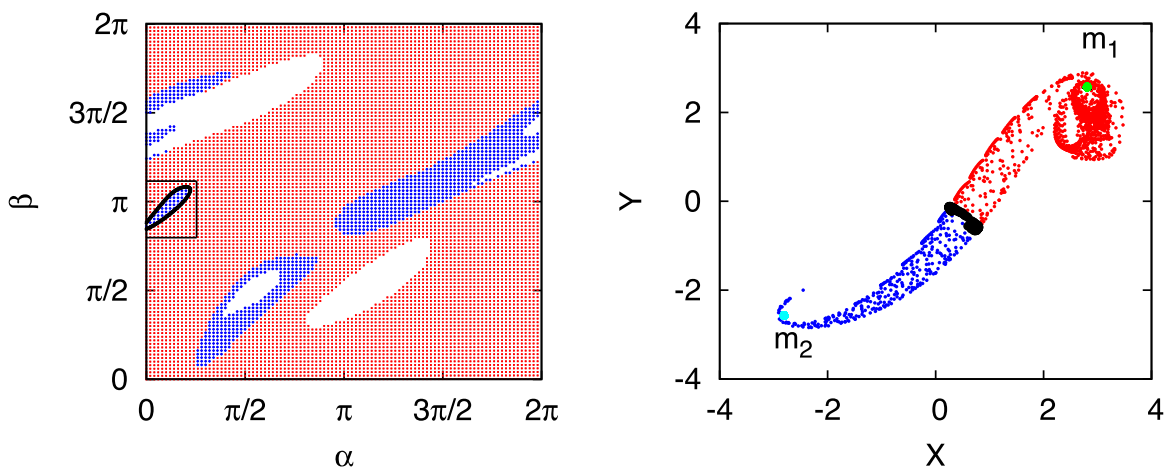

Figure 8. Left: classification plot of $(\alpha, \beta)$ points for $C=8$ and $r_{c}=0.2$. The rectangle shows the region where the initial conditions are taken. Right: snapshot for $\theta=1.2$. Points on the $W^{s}\left(L_{2}^{+}\right)$are also plotted. $(\mu=0.5)$.

left -, which correspond (backwards and forwards in time) to heteroclinic orbits between $m_{1}$ and $L_{3}^{+}$. Close to these heteroclinic orbits, we have escape orbits and orbits such that the particle remains captured by $m_{1}$. This is the mechanism that provokes the apparition of a tail. In Fig. 7, right, we plot a snapshot at $\theta=1.2$ of the location of the particles $[(X, Y)$ in the inertial frame] with initial conditions $(\alpha, \beta)$ at the selected zone (the close encounter between the primaries has already taken place at $\theta=0$ ). We observe the set of particles -red and black colours- that spread on a large region of the $(X, Y)$ plane with the shape of a tail. As time tends to infinity $\left(\theta \rightarrow \frac{\pi}{2}\right)$, some of them must remain around $m_{1}$, others must escape and others must tend to the $L_{3}^{+}$point (the thick curve in the right plot).

Concerning the second zone, the points belonging to the frontier between the two capture regions - blue and red (the thick curve in Fig. 8, left) - correspond (integrating backwards and forwards in time) to heteroclinic orbits between $m_{1}$ and $L_{2}^{+}$. Similar to the previous case, as close to these heteroclinic orbits as desired, we have orbits such that the particle is captured by $m_{2}$ and orbits such that the particle is captured by $m_{1}$, when increasing the time (or $\theta$ ). This is the mechanism that provokes the apparition of a bridge. In Fig. 8, right, we plot a snapshot at $\theta=1.2$ of the location of the particles $[(X, Y)$ in the inertial frame $]$ with initial conditions $(\alpha, \beta)$ at the selected region. We observe the set of particles connecting both primaries with the shape of a bridge. As before, when time tends to infinity $\left(\theta \rightarrow \frac{\pi}{2}\right)$, some of them must remain around $m_{1}$, others must transfer to a neighbourhood of $m_{2}$ and remain around it and others must tend to the $L_{2}^{+}$point (the thick curve in the right plot is close to $L_{2}$ for $\theta=1.2$ ).
To show a bridge and a tail all together, we choose a suitable zone of initial conditions, e.g. $(\alpha, \beta) \in[5,5.5] \times[3,4], r_{c}=0.00405(\simeq$ $1 / 100$ times the maximum $r_{c}$ allowed by the zero velocity curve) and $C=8$. In Fig. 9, we plot the zone in the $(\alpha, \beta)$ plane considered (top left) and three different snapshots of the positions ( $X$, $Y$ ) of each particle at $\theta=0, \theta=\pi / 8$ and $\theta=1.2$. As explained above, the different coloured layers of initial conditions will give rise to bridges and tails. We just remark that due to the red thin layers between the white and blue regions in the classification plot shown in Fig. 9, top left, the tail will be formed behind $m_{1}$, although in Fig. 9, bottom right, it is apparently formed around $m_{2}$. As time increases, for $\theta>1.2$, there will appear two new bridges (corresponding to the two thin red-blue transitions) as well as a tail behind $m_{1}$.

We emphasize that other values of $C \geq C\left(L_{2}\right)=8$ might be taken. We have done the simulations for different values of $C$, ranging from 8 to 10 , and for each fixed $C$, we have varied the value of $r_{c}$ from 0 to 0.2 (or the maximum possible value defined by the bounded Hill region around $m_{1}$ ). We have observed that, as $C$ increases, by (11) the modulus of the velocity for the initial conditions decrease. That means that for bigger values of the initial $C$, the particles have a smaller initial velocity, and also, Hill's region is smaller, so they have a smaller chance to escape. In order to have quantitative estimates of the different types of orbits, we plot in Fig. 10 the proportions of orbits captured by $m_{1}, m_{2}$ and escape with respect to the total of orbits computed, for different values of $C$. As expected, as $C$ increases, a higher (lower) proportion of trajectories remaining captured by $m_{1}$ (escape) is obtained. Nevertheless, we see that still there are orbits escaping or being captured by $m_{2}$. 

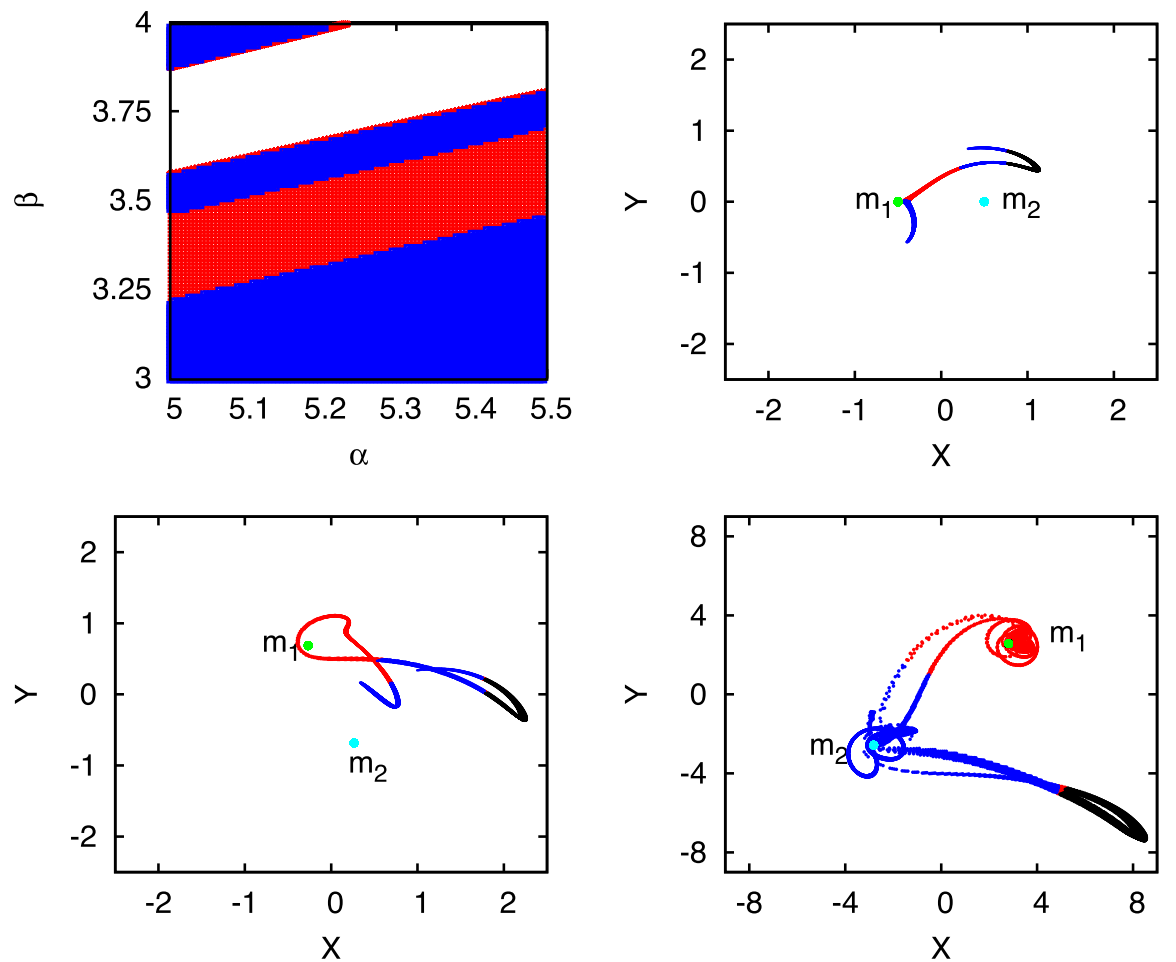

Figure 9. Top left: Detail in the $(\alpha, \beta)$ plane of the classification of the final evolutions of the initial conditions (19), for $C=8$ and $r_{c}=0.00405$. Top right and bottom: Positions in the inertial plane $(X, Y)$ of a set of particles with initial conditions that correspond to orbits escaping, captured by $m_{1}$ or captured by $m_{2}$. The snapshots correspond to $\theta=0$ (top right) and $\theta=\pi / 8$ and $\theta=1.2$ (bottom, respectively).

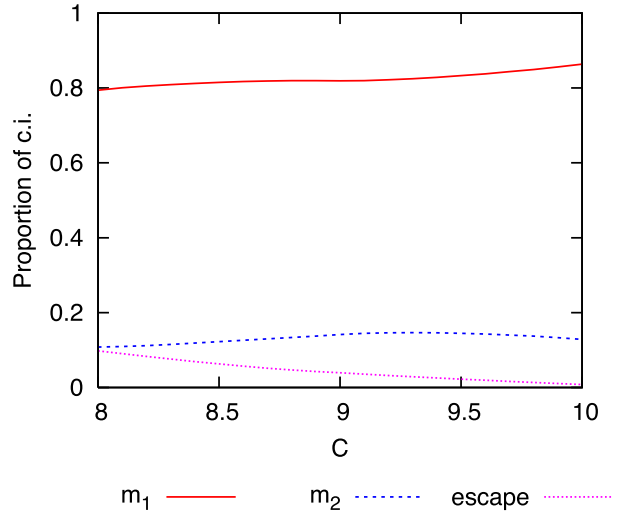

Figure 10. Proportions of orbits captured by $m_{1}, m_{2}$ and escape with respect to the total of orbits computed, for different values of $C$ and $\mu=0.5$.

\subsection{Results for different masses}

Although the model considered is academic, we want to consider the case of galaxies of different mass that corresponds to consider the primaries with different masses, i.e. $\mu<0.5$. We do not intend to take particular values of the mass parameter, associated with real pairs of interacting galaxies, but simply we want to check that the mechanism that explains the apparition of tails and bridges also applies for any value of $\mu$. We have taken a set of test particles around both primaries as in (19) for different values of $\mu$ and we have encountered heteroclinic orbits connecting the primaries $m_{j}$, $j=1,2$ with the collinear equilibrium points. The distinction with the case $\mu=0.5$ is that the primaries have different masses, so we have to consider the influence of each primary separately and compute the corresponding proportions of orbits that tend to capture to each primary or escape.

Just as an illustration of the simulations done, in Fig. 11 we show a snapshot for $\mu=0.3$ where a bridge is clearly apparent, and a snapshot for $\mu=0.1$ to show a bridge and a tail. The initial conditions have been taken similarly as in Figs 7 and 8: once the classification plots for $\mu=0.3$ and $\mu=0.1$ are obtained (not included here), we take initial conditions from regions with different colours, so that bridges and tails can be obtained. More precisely, the left plot is obtained using $\mu=0.3$, with initial conditions starting in a circumference of radius 0.01 around the big primary, and $\alpha \in$ $[1.2,2.2], \beta \in[0.25,1.75]$. The initial conditions for the right plot depart from the small primary with $\mu=0.1$ and $\alpha \in[0.8,1]$ and $\beta$ $\in[3.6,3.9]$.

Following the procedure to obtain Fig. 10, we have also computed the proportions of orbits captured by $m_{1}, m_{2}$ and escape with respect to the total of orbits computed, for different values of $C$ (ranging in the interval $\left.\left[C\left(L_{2}\right), 10\right]\right)$ for different values of $r_{c}$ and different values of $\mu$. In Fig. 12, we show the proportions of initial conditions of each type as $C$ varies, for the values of $\mu=0.5,0.3,0.1$. Since $C\left(L_{2}\right)$ varies with $\mu$, we consider the normalized value $C / C\left(L_{2}\right)$. In order to compare the variation for different values of $\mu$, we show the proportion of orbits captured by $m_{1}$ in the left plot, the proportion of orbits captured by $m_{2}$ in the centre plot, and the proportion of orbits that escapes in the right plot. These proportions take into account if the particles are in a neighbourhood of $m_{1}$ or $m_{2}$ before the close passage of the primaries (i.e. at the initial conditions for $\theta=-\pi / 4$ ) - we will say that the particles leave $m_{1}$ or $m_{2}$, and of course, backward in time, they collide with $m_{1}$ or $m_{2}-$. The solid and dashed lines correspond to orbits that leave $m_{1}$ and $m_{2}$, respectively. The colours orange, violet and green correspond to $\mu=0.5, \mu=0.3$ and $\mu=0.1$, respectively (labels in the plots have been added for black-white print). 

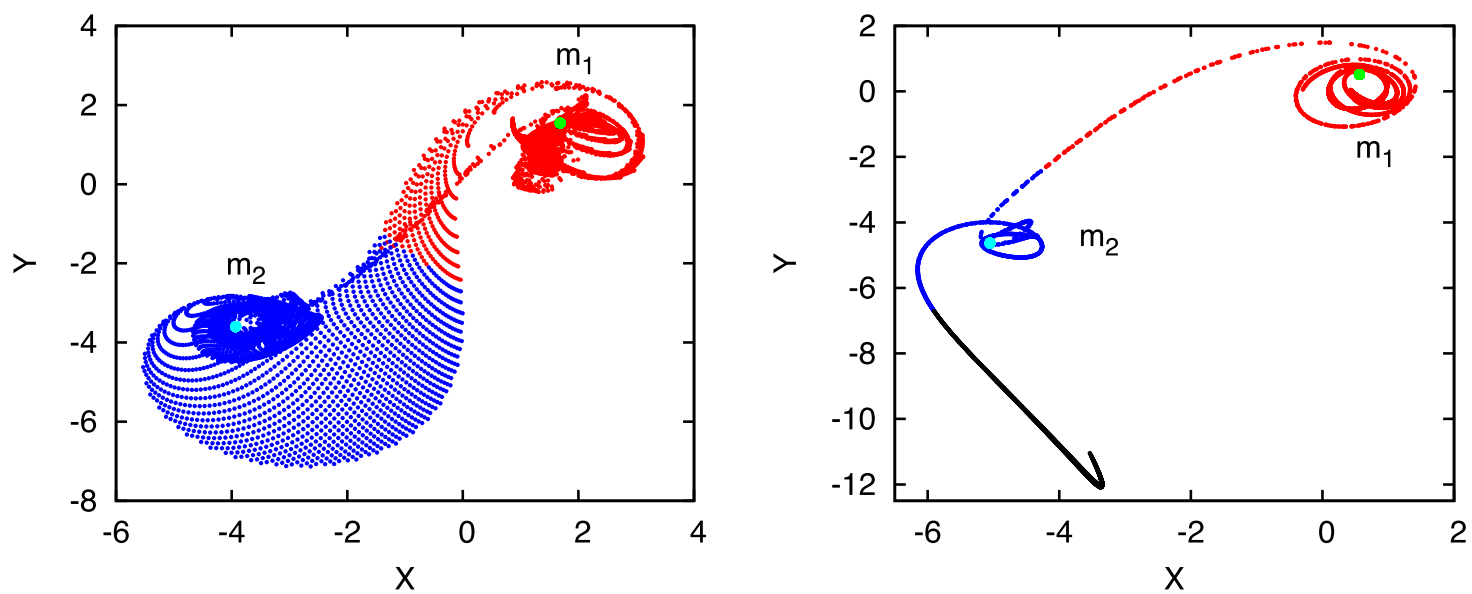

Figure 11. Snapshot to show a bridge for $\mu=0.3$ (left) and both a bridge and a tail for $\mu=0.1$ (right).
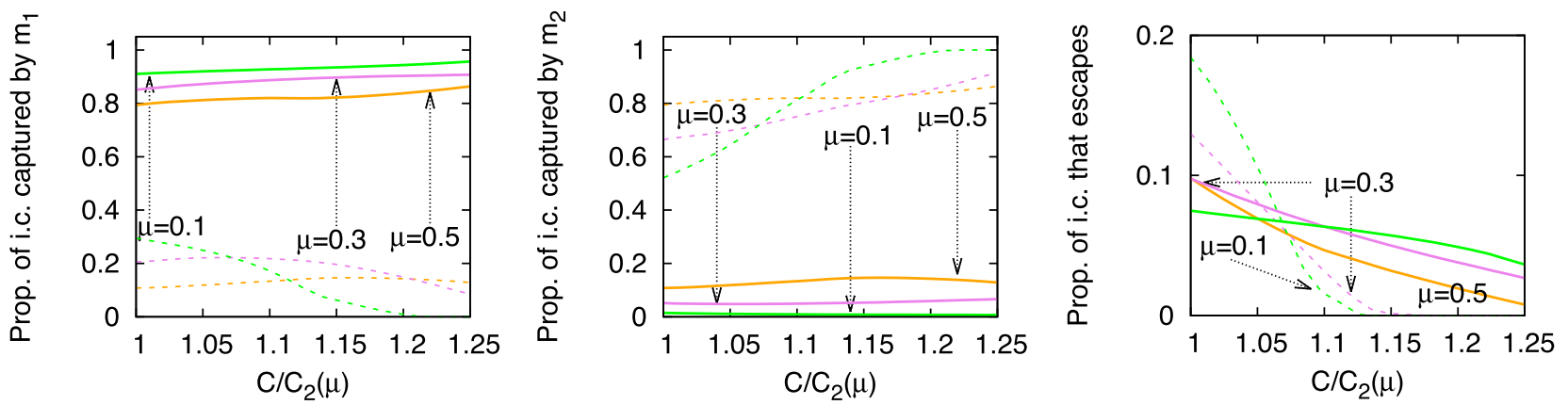

Figure 12. Proportions of initial conditions (i.c.) captured by $m_{1}$ (left), $m_{2}$ (centre) and escape (right) with respect to the total of orbits computed, for different values of $C$ and $\mu=0.5$ (orange), $\mu=0.3$ (violet) and $\mu=0.1$ (green). Solid/dashed lines correspond to orbits leaving a neighbourhood of $m_{1} / m_{2}$ respectively.

As we can see from the left plot, for $C$ close to (and bigger than) $C\left(L_{2}\right)$, the smaller the value of $\mu$, the bigger the number of orbits captured by $m_{1}$ (assuming that the particles leave $m_{1}$ or $m_{2}$ ). However, when the particles leave $m_{2}$, the tendency in the proportion of capture orbits by $m_{1}$ is inverted when $C$ increases, which seems reasonable because with bigger values of $C$, Hill's region around $m_{2}$ shrinks with decreasing $\mu$ and there is a less possibility to leave the neighbourhood of $m_{2}$.

From the centre plot, an opposite behaviour can be observed for the proportion of capture orbits by $m_{2}$. For $C$ close to (and bigger than) $C\left(L_{2}\right)$, this proportion decreases with $\mu$ (when leaving $m_{1}$ or $m_{2}$ ), but the tendency is inverted when $C$ increases, i.e. the proportion increases with $\mu$, just taking into account the particles that leave $m_{2}$.

Finally, taking into account both proportions of capture by the primaries, we obtain the right plot for the escape orbits. We observe, in particular, that given $\mu$ and for (suitable) big values of $C$, there are no escape orbits.

\section{DISCUSSION}

At this point, it seems appropriate to discuss how our results compare with previous ones in the literature.

As mentioned in the Introduction section, several papers analyse the observed bridges and tails for pairs of interacting galaxies. These papers use different models and a first topic in this Discussion section is related to the model we have considered, the parabolic RTBP. Two remarks must be done concerning this model: (i) we assume that each galaxy is modelled as a disc of non-interacting particles orbiting around a central mass point (a primary), whereas the two mass points move in parabolic orbits with respect to their centre of mass. In particular, trajectories described by any particle that passes very close to a primary or even collides with it may take place. A regularization of the system of ODE must be carried out as an strategy to deal with the numerical integration of the system close to or at a singularity (collision with a primary). (ii) Of course, the parabolic RTBP is a very simple model, but we emphasize that our purpose in this paper is to show a mechanism, using dynamical systems tools and more particularly using invariant manifolds, that explains the formation of tails and bridges in this simple model.

As a second topic, and concerning the references mentioned in the Introduction section, we will focus on Toomre \& Toomre (1972) paper, since our interest for the problem starts with their work, where the authors show that bridges and tails appear when the encounter of two galaxies is also modelled by the parabolic problem.

The authors consider two situations: equal masses (which corresponds to $\mu=0.5$ ) and when the big primary is four times bigger than the small one (i.e. $\mu=0.2$ ). Once $\mu$ is fixed, they take a bunch of particles - located in different rings - around one primary (typically the big one) and far from the other primary, which is bare. In this situation, the dynamics around the primary can be modelled by a two-body problem (the influence of the second primary is a perturbation), and the particles are assumed to be in circular orbits around the primary in direct or retrograde motion. Then, the trajectories of all the particles are followed and their location in the inertial frame is plotted at different instants (what we have called snapshots). The relevant point to observe is how these snapshots evolve with time and more particularly, what is the effect 

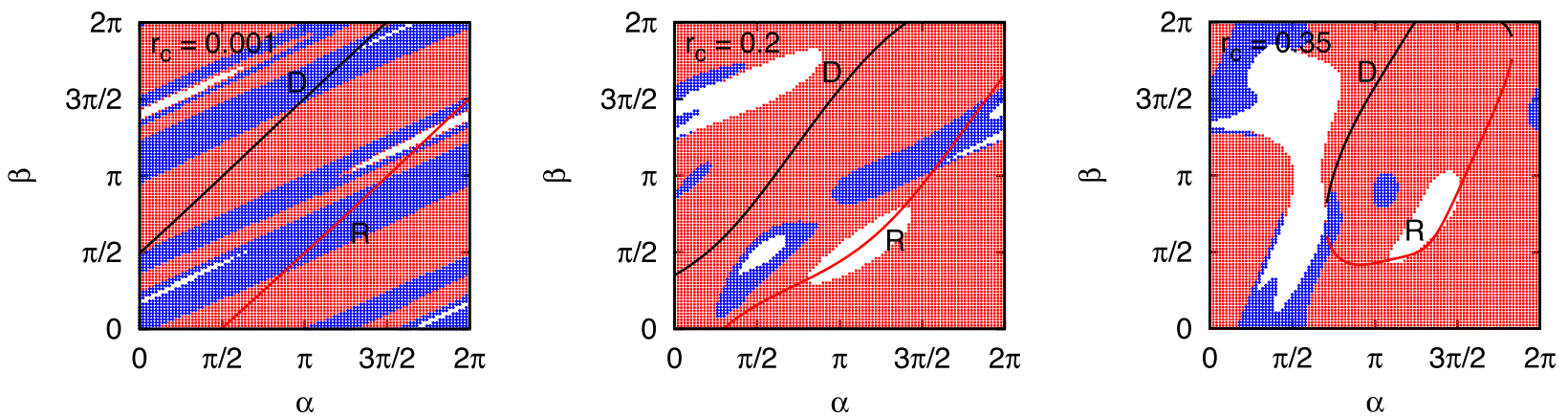

Figure 13. Classification plots of the method $\mathrm{TT}_{1}$ in the $(\alpha, \beta)$ plane for $C=8$ and $r_{c}=0.001, r_{c}=0.2$ and $r_{c}=0.35$ (from left to right) and the curves $\beta_{1,2}$ (D stands for direct orbits, $\mathrm{R}$ for retrograde orbits). $\mu=0.5$.

of the close encounter between the galaxies (primaries). Although Toomre and Toomre show some snapshots where bridges and tails are apparent, they do not give a dynamical explanation on their formation.

We have seen that a dynamical explanation for bridges and tails comes from the existence of heteroclinic connections between collision with the primaries and the equilibrium points $L_{i}^{+}, i=1,2$, 3 . Here, we want to reproduce some explorations of Toomre and Toomre's paper in order to show that the initial conditions that they considered are close to these heteroclinic connections, so this is the reason why they see bridges and tails.

More precisely, we perform the explorations in two ways:

(i) Method $1\left(\mathrm{TT}_{1}\right)$ : we recall that in the Section 3, we have obtained the classification plots (Figs 6,7 and 8), considering all the possible initial conditions in position (varying the synodical radius $r_{c}$ ) and velocity for a given $C$. In this first simulation, we want to restrict, among this whole set of initial conditions, to those synodical initial conditions $\left(x, y, x^{\prime}, y^{\prime}\right)$ as in (19), at $\theta=-\pi / 4$, such that the sidereal velocity $\left(X^{\prime}, Y^{\prime}\right)$ is perpendicular to the position vector $(X, Y)$, centred at the big primary. To satisfy this restriction, for each value of $\alpha$, only two values of $\beta$ are admissible:

$\beta_{1,2}(\alpha)=\alpha \pm \arccos \left(\frac{\sqrt{2}}{v}\left(\mu(\sin \alpha+\cos \alpha)+r_{c}\right)\right)$,

where the \pm sign corresponds to a direct/retrograde orbit.

This means that the initial conditions are at the apoapsis or periapsis of their orbits around the primary, although we do not ensure that the orbit is circular (thinking in a two-body problem $m_{1}$ plus a particle) because the modulus of the synodic velocity $v$ is obtained from the fixed value of $C$. Although this method $\mathrm{TT}_{1}$ is not exactly the one carried out by Toomre and Toomre, in the sense that the initial conditions taken by our method are not necessarily in circular orbits, the exploration done in this way allows us to compare the results obtained with the ones previously shown in Section 3, because the initial value of $C$ is fixed. So, with this method, we obtain the curves $\beta_{1,2}(\alpha)$ once $\mu, r_{c}$ and $C$ are fixed and the simulation aims at checking if such curves overlap different coloured regions when the classification plots are considered.

Concerning the results, we take $\mu=0.5$ and $C=8$, varying $r_{c}$ and $\alpha \in[0,2 \pi]$ (other values of $C$ might be taken, but the same kind of results are obtained). In Fig. 13, we show the classification plot for all values of $\alpha$ and $\beta$ (see also Fig. 6) and the curves $\beta_{1,2}$. The key point to remark about this Figure is that the curves computed overlap regions of different colour, i.e. there are orbits that will collide with $m_{1}$ (with initial conditions provided by points on the curve and on the red region), or will collide with $m_{2}$ (on the blue one) or that escape (white one). Therefore, any point along the curves $\beta_{12}$ that belongs to the frontier of two different coloured regions gives rise to an initial condition of a heteroclinic connection between collision with $m_{1}$ and a collinear equilibrium point. So, following the mechanism described, taking initial conditions along one of the curves and such that there is a transition in colour redblue (or red-white or blue-white), bridges (tails) appear (for that fixed value of $r_{c}$ ).

Another relevant comment is that when $r_{c}$ increases, the direct orbits overlap only the red region, i.e. all the orbits remain captured by $m_{1}$; see Fig. 13 middle and right. For the retrograde case, the curve overlaps different coloured regions, so tails and bridges are expected in this case. This is in accordance with Toomre and Toomre's simulations: roughly speaking, for big values of $r_{c}$, retrograde initial conditions give rise to tails and bridges but direct ones do not.

(ii) Method $2\left(\mathrm{TT}_{2}\right)$ : we fix a value of $\mu$ and a primary, $m_{j}, j=1$, 2. At $\theta_{0}=-\pi / 4$, we take two sets of sidereal initial conditions in rings of radius $R_{c}$ centred at the primary, i.e. $\left(X, Y, X^{\prime}, Y^{\prime}\right)$ as:

$\begin{aligned} X & =X_{m_{j}}+R_{c} \cos \bar{\alpha}, & X^{\prime} & =V \cos \bar{\beta}, \\ Y & =Y_{m_{j}}+R_{c} \sin \bar{\alpha}, & Y^{\prime} & =V \sin \bar{\beta},\end{aligned}$

such that $V^{2}=(1-\mu) / R_{c}$ for $j=1$ or, i.e. velocity to ensure initial conditions on a circular orbit and $\bar{\beta}=\bar{\alpha} \pm \pi / 2$ [so the position with respect to the primary $\left(X_{m_{j}}, Y_{m_{j}}\right)$ and the velocity vectors is perpendicular]; as before, the \pm sign refers to a direct/retrograde orbit, called from now on direct and retrograde cases, respectively.

For each sidereal initial condition, we compute the corresponding synodic initial condition $\left(x, y, x^{\prime}, y^{\prime}\right)$ that can be written as in (19) with $r_{c}=R_{c} / 2$, although now all of them have a different value of $C$ (and we have checked that all the particles remain captured by the primary $m_{j}$ backwards in time). The orbits are followed until they are classified depending on their future evolution. Then we plot the initial conditions $(X, Y)$ coloured as follows: red if the orbit collides with $m_{1}$, blue if collides with $m_{2}$ and green if it escapes.

We want to study the contribution to tails and bridges of particles starting around each primary. Thus, we consider separately initial conditions around $m_{1}$ or $m_{2}$, and direct and retrograde orbits, for different values of $\mu$. In each case, we show the results for the values of $\mu$ for which there are significant differences in order to see the evolution of the sets of capture orbits and escape orbits as $\mu$ decreases.

Concerning the results obtained taking rings of particles around the big primary $m_{1}$, in Fig. 14 we show the classification plots for the direct case. We see that only collision orbits with $m_{1}$ and escape orbits appear, and that as $\mu$ decreases, all the initial conditions 

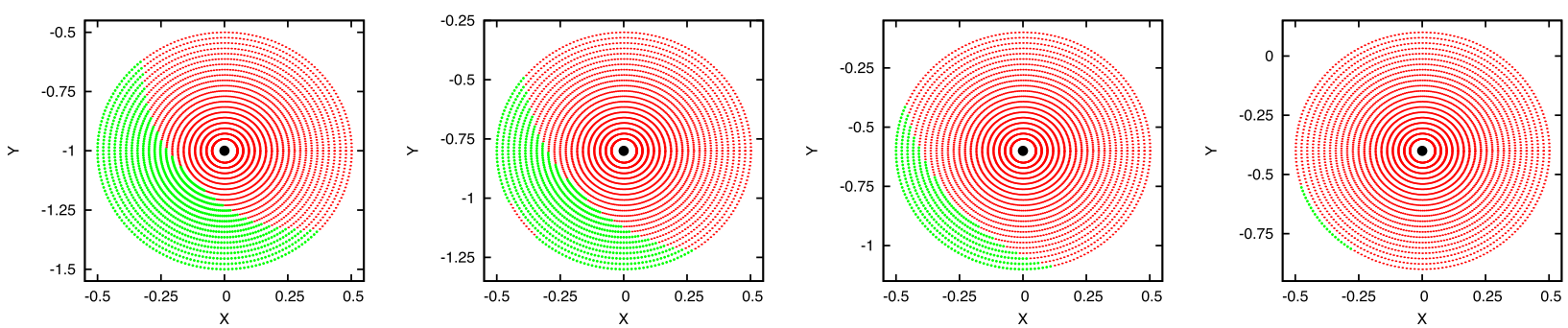

Figure 14. Results of the method $\mathrm{TT}_{2}$. Initial conditions $(X, Y)$ for direct orbits leaving a neighbourhood of $m_{1}$ at $\theta_{0}=-\pi / 4$, classified depending on their final evolution. Only capture orbits to $m_{1}$ (red, light grey) and escape orbits (green, medium grey) are obtained. From left to right: $\mu=0.5, \mu=0.4, \mu=0.3$ and $\mu=0.2$.
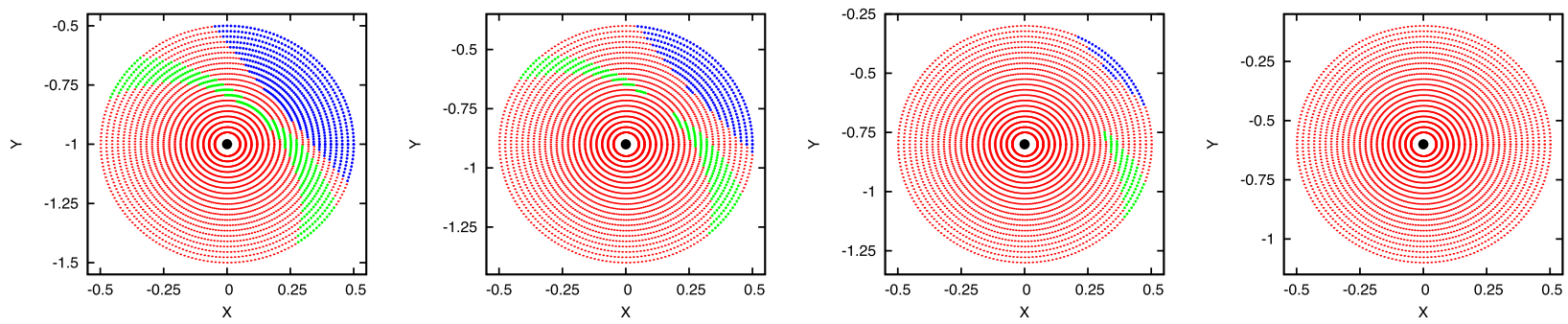

Figure 15. Results of the method $\mathrm{TT}_{2}$. Initial conditions $(X, Y)$ for retrograde orbits leaving a neighbourhood of $m_{1}$ at $\theta_{0}=-\pi / 4$, classified depending on their final evolution (capture $m_{1}$ : red, light grey; escape: green, medium grey; capture $m_{2}$ : blue, dark grey). From left to right: $\mu=0.5, \mu=0.45, \mu=0.4$ and $\mu=0.3$.

correspond to collision orbits. Recall that the smaller the value of $\mu$, the bigger the mass of $m_{1}$. Therefore, direct orbits only contribute to the formation of tails if $\mu$ is not too small. Regarding the retrograde case, we observe from Fig. 15 that there appear regions of the three different colours for bigger values of $\mu$ and only capture by $m_{1}$ for moderate values of $\mu$. So tails and bridges will be observed just for big values of $\mu$.

Comparing our results with Toomre and Toomre's paper, several remarks must be done:

(i) Regarding the $\mu=0.5$ case (equal masses), for the direct case, the authors in their paper do not observe tails because they consider a too short range of integration time, whereas in our simulations all the orbits are classified (integrating enough time), and we can observe that there are orbits with two different final evolutions: capture by $m_{1}$ (red) and escape (green) so that the formation of tail is guaranteed. With respect the retrograde case, we do agree with them concluding that retrograde initial particles provide both tails and bridges.

(ii) Taking into account different mass galaxies, and initial rings of particles around $m_{1}$, Toomre and Toomre just consider two cases: $\mu=0.5$ and $\mu=0.2$, but in the latter, they examine only the retrograde case. In this paper, we have taken several different values of $\mu \in(0,0.5]$ and also both the direct and retrograde cases. We can conclude from the plots in Figs14 and 15 that tails are obtained for both direct and retrograde cases, assuming big values of $\mu$ (for $\mu$ $\leq 0.2$ only capture by $m_{1}$ is obtained); however, bridges do appear only in the retrograde case and also for big values of $\mu$. This is in accordance with their results. At this point, we must remark that Toomre and Toomre do also find bridges for $\mu=0.2$ and retrograde case. But they emphasize that they appear due to big enough values of $R_{c}$ (more particularly, from $R_{c}=0.6,0.7$ ). Of course, all these simulations depend on both the initial value of $\theta(-\pi / 4$ in our simulations and a smaller value in theirs) and on the radius of the rings of particles (we take $R_{c} \in(0.05,0.5]$ and they consider $R_{c}$ $\in(0.2,0.7])$. We do not take bigger values of $R_{c}$ because our goal was to show the role of the invariant manifolds as a mechanism to explain bridges and tails, taking rings of particles close to the primary (and assumed to be captured by the primary backwards in time), whereas in their paper, they do not consider dynamical systems theory but simulate the bridges and tails playing with $R_{c}$ as a parameter.

(iii) A comment to be done is that in Toomre and Toomre's paper, the plots in the first four figures are obtained taking 12, 18, 24, 30, 36,42 particles in each ring of radius $0.1,0.2,0.3,0.4,0.5,0.6$ and 0.7 , respectively. So, they typically say that the bridges are too transient or just observable for a thin period of time and that good bridges do not arise if the two primaries are roughly equal. With our classification criterion, we are able to see bridges for a long time just taking a big enough number of initial particles (actually we took typically 100 particles at each ring and radius up to 0.5 ). And we conclude that there are good (clearly visible) bridges even for equal masses.

Concerning the results obtained taking initial rings of particles around the small primary $m_{2}$, in Figs 16 and 17, we show the classification plots for direct and retrograde orbits, respectively. In the case $\mu=0.5$, we obtain the symmetric plot of Figs 14 and 15 top left, due to the symmetry of the problem. As $\mu$ decreases, more diversity appears in both direct and retrograde orbits, and, in particular, the set of orbits that tend to $m_{1}$ grows. This can be explained simply by the fact that as $\mu$ decreases, $m_{1}$ gets bigger. It is worth mentioning that in Toomre and Toomre's simulations, they assert that for $\mu=0.2$ (retrograde case) - the only case they consider - there do not appear enduring bridges. However, as we have seen from our simulations, for $\mu \leq 0.3$, in the direct case and for all $\mu \in(0,0.5]$ in the retrograde one, there do appear bridges, since coloured red-blue regions are obtained; see Figs 16 and 17.

A final comment is that both in Toomre and Toomre's paper and in method 2 of our computations, only initial rings assumed to 

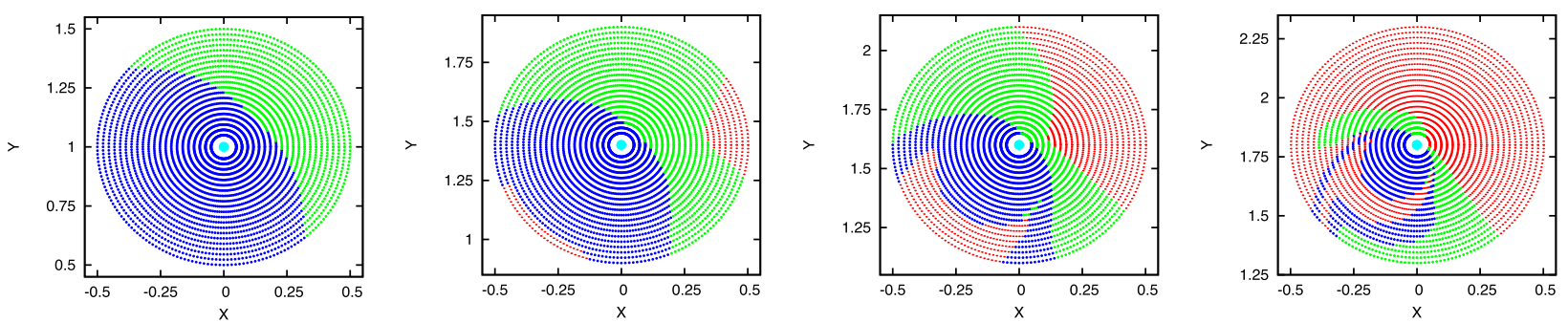

Figure 16. Results of the method $\mathrm{TT}_{2}$. Initial conditions $(X, Y)$ for direct orbits leaving a neighbourhood of $m_{2}$ at $\theta_{0}=-\pi / 4$, classified depending on their final evolution (capture $m_{1}$ : red, light grey; escape: green, medium grey; capture $m_{2}$ : blue, dark grey). From left to right: $\mu=0.5, \mu=0.3, \mu=0.2$ and $\mu=0.1$.
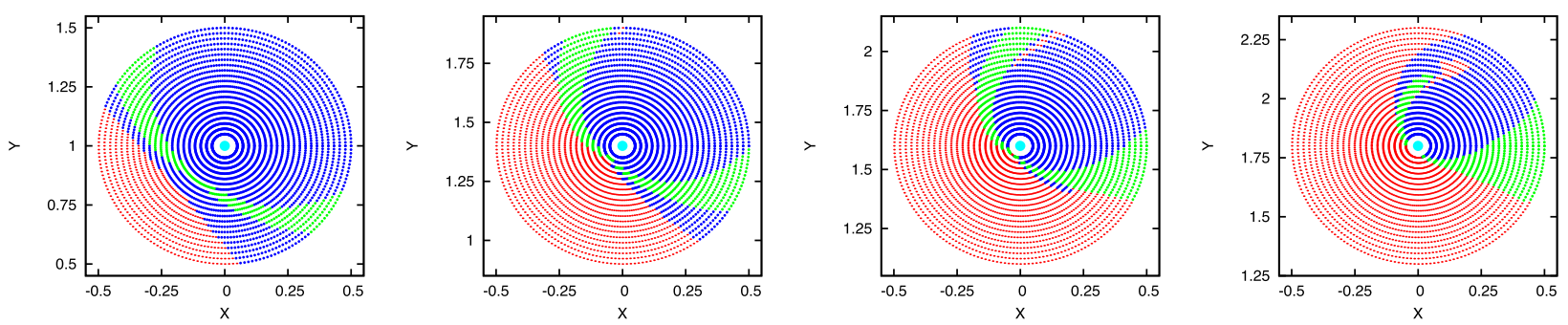

Figure 17. Results of the method $\mathrm{TT}_{2}$. Initial conditions $(X, Y)$ for retrograde orbits leaving a neighbourhood of $m_{2}$ at $\theta_{0}=-\pi / 4$, classified depending on their final evolution (capture $m_{1}$ : red, light grey; escape: green, medium grey; capture $m_{2}$ : blue, dark grey). From left to right: $\mu=0.5, \mu=0.3, \mu=0.2$ and $\mu=0.1$.

describe circular orbits are considered. Of course, other effects as eccentricity and inclination might be taken into account (actually they consider inclination and we do not). For realistic models, of course, such effects are relevant, but we insist that in this paper, the purpose was to present a mechanism that explains the formation of bridges and tails, using dynamical systems tools and a very simple model. The next natural step is to take other more realistic models and compare and contrast the new simulations obtained (concerning bridges and tails) with the morphology of particular observed real interacting galaxies. But this will be the goal of a future paper.

\section{CONCLUSIONS}

Although some observations are published showing the existence of bridges and tails resulting from interacting galaxies, as far as we know, this is the first attempt to explain their formation applying invariant manifolds.

In this paper, we have considered a very simple model, the planar parabolic RTBP, where each galaxy is modelled as a disc of noninteracting particles orbiting around a central mass point (a primary) whereas the two primaries move in parabolic orbits with respect to their centre of mass. A regularization of the system of ODE has been carried out in order to numerically integrate this system allowing close passages or even collisions between a particle and the central mass.

A dynamical mechanism that explains the formation of bridges and tails after a close approach of the two galaxies is analysed. Such an explanation is due to the existence of heteroclinic orbits between the collinear equilibrium points and the collision manifold associated with the primaries. The fact that such invariant manifolds separate different final dynamical evolutions is directly responsible for the formation of tails and bridges. We have done some numerical simulations where the tails and bridges are clearly seen. In addition, we have classified that whole sets of initial conditions for particles (located in rings) that were orbiting one primary, in a direct or retrograde direction, and after a close encounter between the two galaxies, may remain captured by the same primary, or jump and remain captured by the other one or escape.

Roughly speaking, we can conclude that, on one hand, tails are formed either from particles that were orbiting the small primary or from those orbiting the big one if the mass parameter $\mu$ is big. On the other hand, bridges are formed either from particles that were orbiting the small primary or from those orbiting the big one in a retrograde direction for big values of $\mu$.

Finally, the mechanism described has been applied to explain previous simulations on the formation of bridges and tails by other authors, more precisely by Toomre \& Toomre (1972). We have repeated their simulations, drawing the same conclusions. However, the progress with respect to their paper is threefold: (i) we have taken a big number of particles and have classified any trajectory. This approach allows to see permanent bridges and tails (not only transient ones as in their paper). (ii) We have discussed the presence of bridges and tails for different values of the mass parameter, and assuming that the rings of particles surround initially one or another primaries. (iii) We have analysed why the suitable invariant manifolds explain the formation of bridges and tails.

\section{ACKNOWLEDGEMENTS}

E. Barrabés has been supported by grants MTM2013-41168-P and MTM2016-80117-P (MINECO/FEDER, UE), and AGAUR grant SGR1145. J.M. Cors has been supported by MINECO/FEDER grant MTM2013-40998-P, MTM2016-77278-P and AGAUR grant 2014 SGR 568. L. Garcia-Taberner and M. Ollé have been supported by the Spanish MINECO/FEDER grant MTM2015-65715-P and the Catalan grant 2014SGR-00504.

\section{REFERENCES}

Alvarez M., Cors J. M., Delgado J., 2006, Celestial Mech. Dynam. Astronom., 95,173 
Athanassoula E., Romero-Gómez M., Masdemont J., 2009a, Mon. Not. R. Astron. Soc., 394, 67

Athanassoula E., Romero-Gómez M., Masdemont J., 2009b, Mon. Not. R. Astron. Soc., 400, 1706

Barrabés E., Cors J., Ollé M., 2015, Commun. Nonlinear Sci. Numer. Simul., 29,400

Belokurov V., Erkal D., Deason A. J., Koposov S. E., De Angeli F., Evans D. W., Fraternali F., Mackkey D., 2017, MNRAS, 466, 4711

Condon J., Helou G., Sanders D., Soifer B., 1993, AJ, 105, 1730

Danby J., 1992, Fundamentals of Celestial Mechanics, 2nd edn. WillmannBell, Inc., Richmond, USA

Faintich M., 1972, Celest. Mech. Dyn. Astro., 6, 22

Fragner M., Nelson R., 2009, A\&A, 505, 873

Günthardt G., Agüero E., Rodrigues I., Díaz R., 2006, A\&A, 453, 801

Meyer K. R., Hall G. R., 1992, Introduction to Hamiltonian Dynamical Systems and the $N$-Body Problem, Applied Mathematical Sciences Vol. 90. Springer-Verlag, New York
Namboodiri P., Kochhar R., Alladin S., 1987, Bull. Astr. Soc. India, 15, 186 Pfalzner S., Vogel P., Scharwächter J., Olczak C., 2005, A\&A, 437, 967

Pinyol C., 1995, Celestial Mech. Dynam. Astronom., 61, 315

Romero-Gómez M., Masdemont J., Athanassoula E., García-Gómez C., 2006, A\&A, 453, 39

Steinhausen M., Olczak C., Pfalzner S., 2012, A\&A, 538

Szebehely V., 1967, Theory of Orbits. The Restricted Problem of Three Bodies. Academic Press, Inc., New York

Toomre A., Toomre J., 1972, ApJ, 178, 623

Tsoutsis P., Kalapotharakos C., Efthymiopoulos C., Contopoulos G., 2009, A\&A, 495, 743

This paper has been typeset from a $\mathrm{T}_{\mathrm{E}} \mathrm{X} / \mathrm{LAT}_{\mathrm{E}} \mathrm{X}$ file prepared by the author. 